\title{
Overview of Recent and Holocene ostracods (Crustacea) from brackish and marine environments of Portugal
}

\author{
M. CRISTINA CABRAL* \& ISABEL M. LOUREIRO \\ Universidade de Lisboa, Faculdade de Ciências, Departamento e Centro de Geologia, Campo Grande, C6, 4º, 1749-016 Lisboa, Portugal \\ *Corresponding author (e-mail: mccabral@fc.ul.pt)
}

\begin{abstract}
An extensive overview of Recent and Holocene ostracods from brackish and marine environments of Portugal is presented for the first time. 178 species belonging to 75 genera have been found and all are listed and illustrated with SEM images. From those species only 30 were found alive, most of them living in marginal-marine environments. Some comments on the 30 species found alive and a summary of main results are also presented. The data come from unpublished notes, reports of several research projects, observations on Recent ostracods in sediments from different estuaries of the western coast of Portugal and from the western Algarve continental shelf and slope, Masters theses and published papers. J. Micropalaeontol. 32(2): 135-159, July 2013.
\end{abstract}

KEYWORDS: Ostracoda list, marginal-marine, continental shelf, Quaternary, Portugal

\section{INTRODUCTION}

There are very few papers about the Recent marine and brackish ostracods of mainland Portugal. The first contribution was published by Paulo (1969), where only five species (1 brackish, 4 freshwater) from Castelo do Queijo, Porto were described. Later, Paulo \& Moutinho (1983) listed 33 species, most of them from freshwater habitats. More recently, in Loureiro et al. (2009), ostracods of the Mira estuary were studied, both alive (autochthonous) and transported by tidal currents. Cabral et al. (2011b) is the first reference to the ostracods from the Portuguese continental shelf. In these two latter works some species are figured.

Information about Holocene ostracods has started appearing more recently, with several papers published since 1999: Hindson \& Andrade (1999), Hindson et al. (1999), Cearreta et al. (2003), Cabral et al. (2006, 2011a), Hilbich et al. (2008), van der Schriek et al. (2008), Almeida et al. (2009) and Lord et al. (2011). In all these papers ostracods come from long boreholes taken from marginal-marine sediments; the ostracods are figured only in Cabral et al. (2006) and Lord et al. (2011).

In spite of their specific merits, most of these works lack scanning electron microscope (SEM) illustrations, which would have rendered them much more useful for comparison with ostracods from other Atlantic and Mediterranean coastal regions. In this work we present, for the first time, an overview of the distribution of Recent and Holocene brackish and marine ostracods in Portugal, with full SEM photographic documentation. In addition, gathering a broad dataset in a single survey allows us to make well-supported conclusions on palaeoenvironmental, palaeogeographical and stratigraphical issues. Freshwater ostracods are not considered in this work, though some of the known species live in slightly saline inland and coastal environments. Recently, an overview of these freshwater Portuguese species was published by Martins et al. (2010).

\section{MATERIAL AND METHODS}

The list presented here is based on unpublished notes (MCC), reports of several research projects (MCC), observations of one of the authors (MCC) on Recent ostracods in sediments from different estuaries of western Portugal and from the Algarve continental shelf and slope (south Portugal), Masters degree theses and published papers. We consider here only the papers that have illustrations of the mentioned ostracods, or whose study material has been identified by the authors (Cearreta et al., 2003; Cabral et al., 2006, 2011a, b; Loureiro et al., 2009; Lord et al., 2011). All the unpublished theses were supervised by MCC in the Department of Geology, Faculty of Sciences, University of Lisbon and are archived and accessible in the Central Library of the Faculty of Sciences and in the Library of the Department of Geology.

The taxa have been identified only on the basis of carapaces without soft parts. Most of the Recent marine taxa, from the continental shelf or slope and from the tidal flats of the lower part of the estuaries, were found in fine-grained sediments, as separate valves, therefore limiting knowledge of where they really live today in the areas where they have been found. The material from the continental shelf and slope was frequently abundant, well preserved and represented by several ontogenic stages suggesting, in this case, that it was in situ. The material from the tidal flats was generally represented by rare valves of each species, frequently worn.

The brackish forms from the tidal marshes were generally collected in muddy sediment, and most of them were found alive, as indicated in the list. Only a few brackish forms from the tidal marshes were collected in fine sand or silty sand substrates. The brackish/marine forms from rock tidal pools and from the transect along the Laje River were collected both on algae and on sandy substrate. The marine forms from the continental shelf and slope were collected with a Smith-McIntyre (SMT) grab, in fine sediment patches (sand fraction generally $<50 \%$ ). Forms were identified as alive when containing the soft parts (appendages) and pink staining due to Rose Bengal. The material from the tidal marshes was collected in two different seasons, generally in autumn and spring, very rarely at the beginning of winter and of summer; the material from the continental shelf and slope was collected during winter (January 2008) and spring (June 2008). Salinity and temperature values from tidal flat, low and high marsh sampling points were measured at low tide in the interstitial sediment water. The records were collected up to May 2012.

The taxonomy follows Horne et al. (2002). For the identification of most of the species, Athersuch et al. (1989) and Bonaduce 

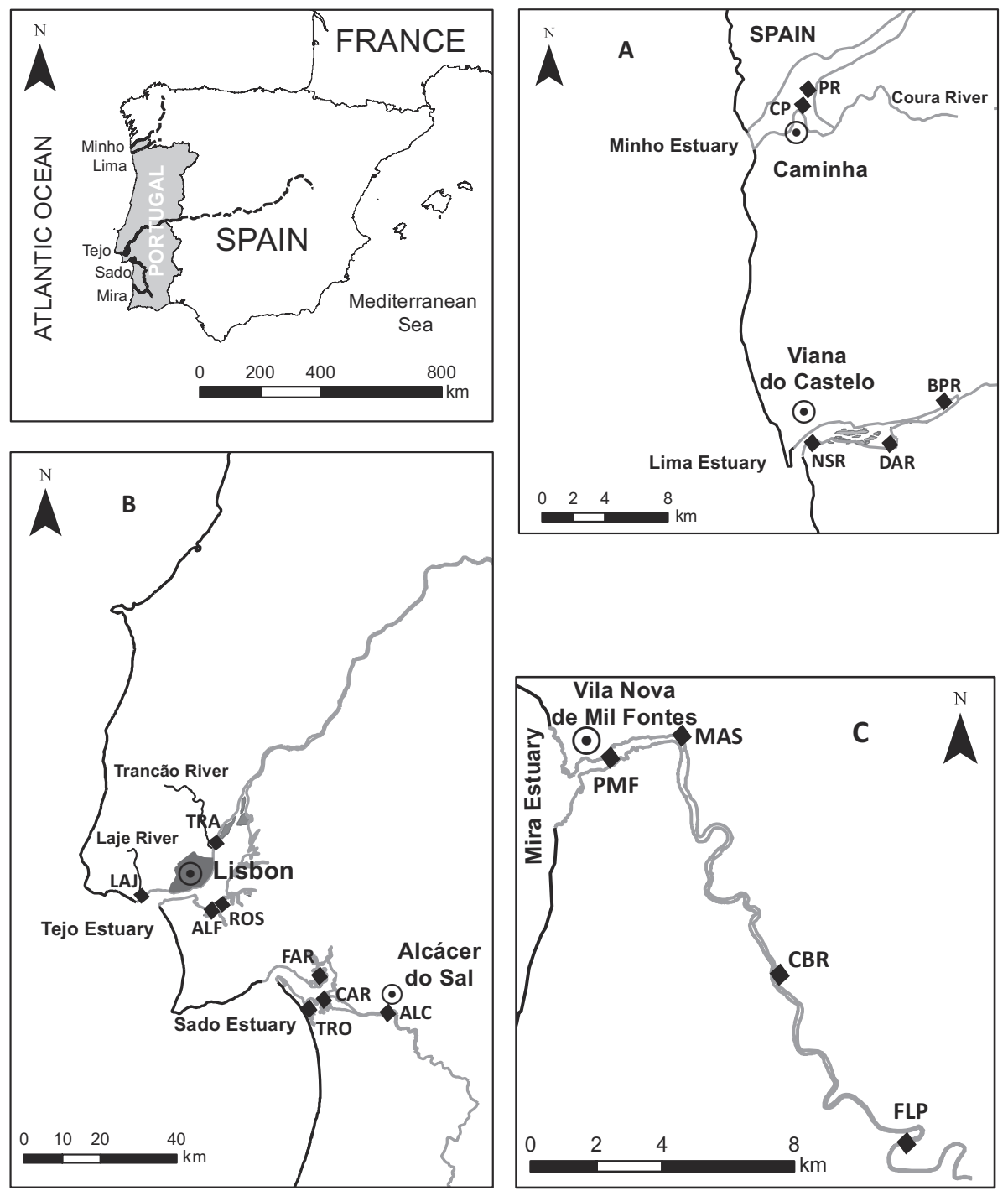

Fig. 1. Location map of the transects in the Portuguese estuaries: A - Minho estuary (CP and PR) and Lima estuary (NSR, DAR, BPR). B - Tejo estuary (LAJ, TRA, ALF and ROS) and Sado estuary (FAR, CAR, TRO and ALC). C - Mira estuary (PMF, MAS, CBR and FLP).

et al. (1976) were used. For some genera or species, several other works were also used: Aiello et al. (1996), Athersuch (1976b), Athersuch \& Horne (1987), Barbeito-Gonzàlez (1971), Breman (1975), Ciampo (1976), Colalongo \& Pasini (1980), Coles et al. (1994, 1996), Horne \& Whittaker (1985), Horne et al. (2004), Lachenal (1989), Schornikov \& Keyser (2004), Whatley \& Masson (1980) and Yassini $(1969,1979)$ and many Stereo-Atlas of Ostracod Shells papers, e.g. Athersuch (1976a, 1981), Athersuch \& Horne (1983), Athersuch \& Whittaker (1982a, b, 1976), Doruk (1974), Guillaume (1988), Horne (1989), Horne \& Robinson (1982, 1985) and Whittaker $(1978 a, b)$.

All the studied specimens are deposited in the Cabral Collection of the Department of Geology, Faculty of Sciences, University of Lisbon.

\section{STUDY AREA}

Recent material comes from several different estuaries of the western Portuguese coast, two in the NW, three in the SW
(Fig. 1) - transects of tidal marsh zones, across the tidal flat, low marsh (Spartina) and high marsh (Salicornia) - and from the Algarve continental shelf and slope (Fig. 2):

Minho River and its Coura tributary - 2 transects, 1 in the Coura confluence with the Minho River (CP), 1 in the lower Minho estuary (PR).

Lima River - 1 transect in the lower estuary (NSR), 2 transects in the mid-estuary (DAR and BPR).

Tejo River and its Trancão and Laje tributaries -2 transects in the lower Tejo estuary (ALF and ROS), 1 transect in the lower Trancão estuary (TRA), $100 \mathrm{~m}$ from the confluence with Tejo (mid Tejo estuary), 1 transect along the lower Laje River estuary (LAJ), from the mouth to $350 \mathrm{~m}$ upriver (lowest part of Tejo estuary/Atlantic ocean) and 2 rock intertidal pools nearby, in Santo Amaro de Oeiras beach.

Sado River -3 transects in the lower estuary (FAR, CAR, TRO), 1 transect in the mid-estuary (ALC). 


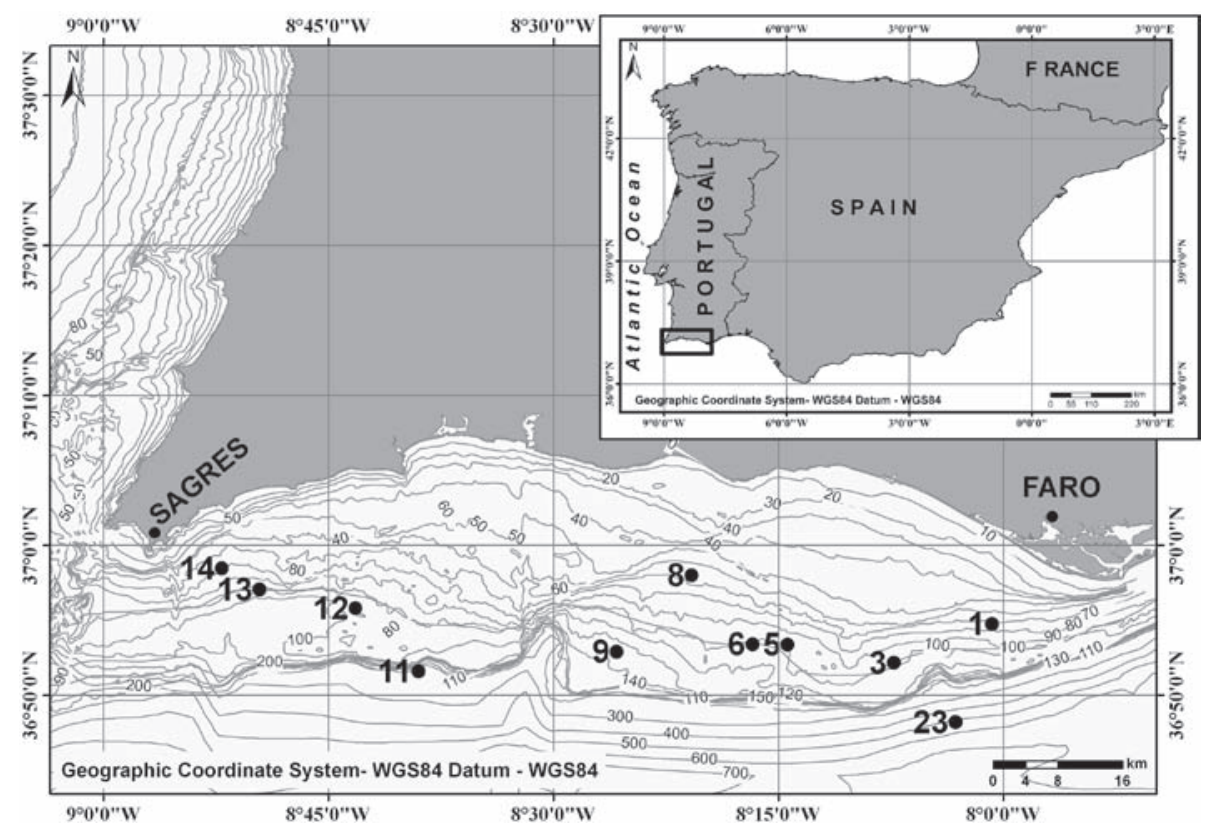

Fig. 2. Location of the studied sites on the western continental shelf and slope of Algarve.

Mira River -2 transects in the lower estuary (PMF and MAS), 2 transects in the mid-estuary (CBR and FLP).

Material also comes from the continental shelf and slope of western Algarve (south Portugal), between Sagres and Faro - 9 sites in the continental shelf $(1,3,5,6,8,9,12,13$ and 14) ranging in depth from -58 to $-125 \mathrm{~m}$ and 2 sites in the continental slope (11 and 23), respectively at c. $-220 \mathrm{~m}$ and $c .-500 \mathrm{~m}$ deep.

Holocene material comes from several boreholes located in coastal lagoons or estuaries (Fig. 3). From north to south:

Pederneira lagoon, west Portugal -2 long boreholes, both in the Recent alluvial plain of Alcoa River, 1 in the inner ancient lagoon (S2), another in the middle ancient lagoon (S3).

Sizandro estuary/lagoon, west Portugal - 1 long borehole in the alluvial plain of Sizandro River, around $10 \mathrm{~km}$ from coast (RS06_1).

Tejo estuary - 1 long borehole in Lisboa central urban area (esteiro da Baixa), Praça do Comércio (PC), and a $4 \mathrm{~m}$ core from a marsh area in Seixal bay (DWK2).

Melides lagoon, SW Portugal - 2 long boreholes, one in the alluvial plain of the Recent lagoon (MIGM), another in the sand barrier (MB).

Santo André lagoon, SW Portugal - 1 long borehole in the alluvial plain of the Recent lagoon (LSA).

\section{LIST OF BRACKISH AND MARINE OSTRACODA FOUND IN PORTUGAL}

Systematics according to Horne et al. (2002). Identifications based primarily on Athersuch et al. (1989) and Bonaduce et al. (1976). Note that primary author sources for individual species have been consulted but, for brevity, are not all listed in the References.

Note: Includes all records, both live forms and dead carapaces/ valves. The localities (generally estuaries) where the Recent forms occurred are ordered from north to south; the localities where the Holocene forms occurred are ordered alphabetically. Species which are referred as cf. compare fairly closely with known species (they present almost all the characteristics of the species, but differ in one or two details, frequently because they are either worn or juveniles); species referred as aff. are probably new, but present some similarities with existing species.

Class Ostracoda Latreille, 1806

Subclass Myodocopa Sars, 1866

Order Halocyprida Dana, 1853

Suborder Cladocopina Sars, 1866

Superfamily Cladocopoidea Sars, 1866

Family Polycopidae Sars, 1866 Genus Polycope Sars, 1866

Polycope reticulata G. W. Müller, 1894 - Mira (Pl. 1, fig. 1)

Subclass Podocopa Sars, 1866

Order Platycopida Sars, 1866

Suborder Platycopina Sars, 1866

Superfamily Cytherelloidea Sars, 1866

Family Cytherellidae Sars, 1866

Genus Cytherella Jones, 1849

Cytherella alvearium Bonaduce, Ciampo \& Masoli, 1976 continental shelf and slope (Pl. 1, fig. 2)

Cytherella robusta Colalongo \& Pasini, 1980 - continental shelf and slope (P1. 1, fig. 3)

Cytherella thrakiensis Stambolidis, 1980 - Mira (P1. 1, fig. 4)

Cytherella cf. vulgata Ruggieri, 1962 - continental shelf (P1. 1, fig. 5)

Genus Cytherelloidea Alexander, 1929

Cytherelloidea cf. sordida (G. W. Müller, 1894) - Holocene Lisboa (P1. 1, fig. 6)

Order Podocopida Sars, 1866

Suborder Cytherocopina Gründel, 1967

Superfamily Cytheroidea Baird, 1850

Family Bythocytheridae Sars, 1866 


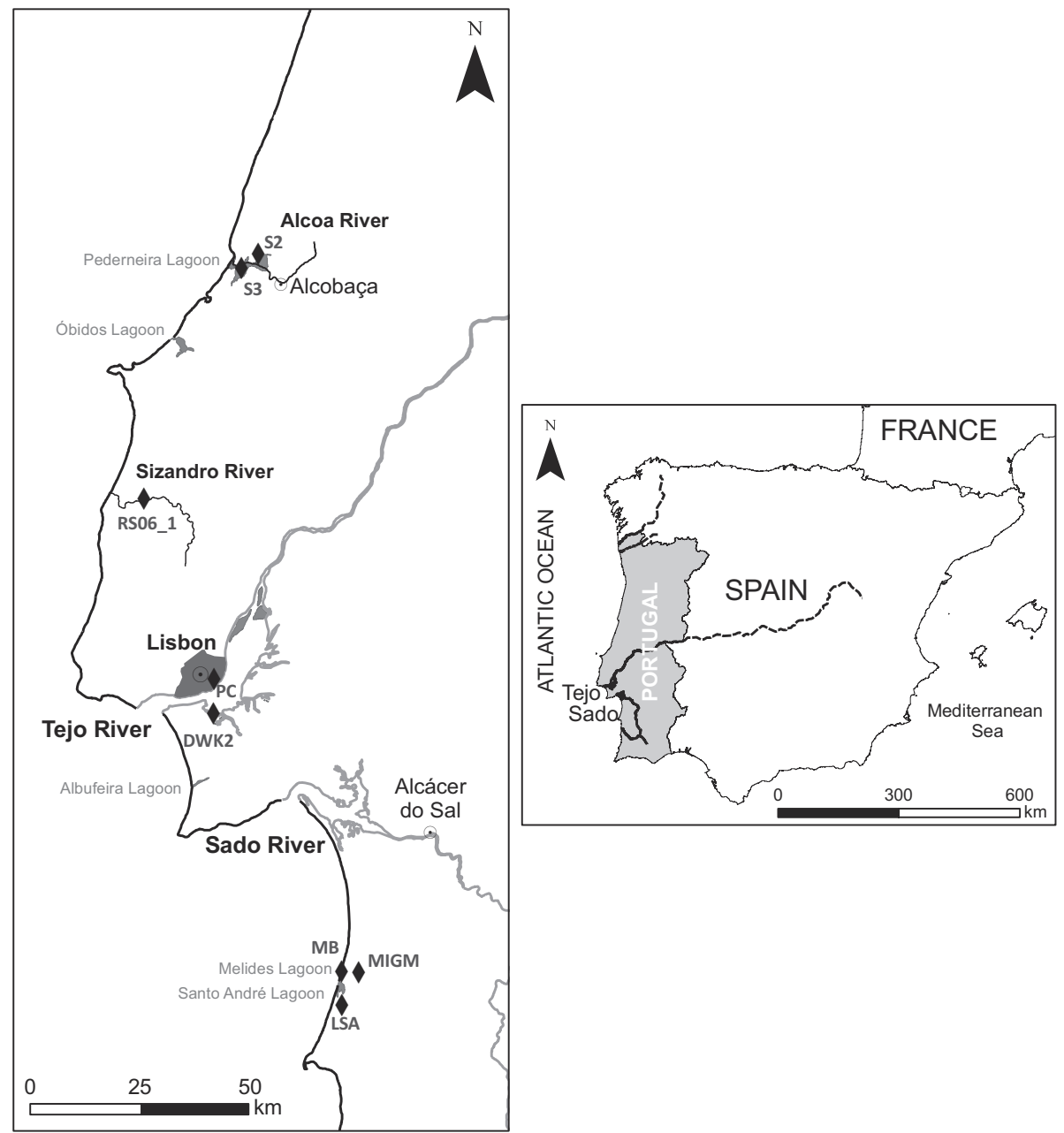

Fig. 3. Location map of the Holocene boreholes: Pederneira lagoon (S3 and S2); Sizandro river (RS06_1); Tejo estuary (PC and DWK2); Melides lagoon (MB and MIGM); Santo André lagoon (LSA).

Genus Bythocythere Sars, 1866

Bythocythere bradyi Sars, 1926 - Mira (P1. 1, fig. 7)

Bythocythere intermedia Elofson, 1938 - Mira, Holocene Lisboa

(P1. 1, fig. 8)

Genus Pseudocythere Sars, 1866

Pseudocythere caudata Sars, 1866 - continental shelf, Mira

(Pl. 1, fig. 9)
Genus Sclerochilus Sars, 1866

Sclerochilus cf. abbreviatus Brady \& Robertson, 1869 - Mira,

Holocene Lisboa (P1. 1, fig. 10)

Sclerochilus gewemuelleri Dubowsky, 1939 - Lima, Mira (P1. 1, fig. 11)

Family Cuneocytheridae Mandelstam, 1959

Explanation of Plate 1. LV, left valve; RV, right valve; C, carapace; f, female; m, male; j, juvenile; aut., autumn; spr., spring. Scale bar $100 \mu \mathrm{m}$. fig. 1. Polycope reticulata G. W. Müller, 1894, LV, external; Mira-PMF, P2 (aut.). fig. 2. Cytherella alvearium Bonaduce, Ciampo \& Masoli, 1976, LV, external; site 12 (POP 0108). fig. 3. Cytherella robusta Colalongo \& Pasini, 1980, LV, external; site 11 (POP 0108). fig. 4. Cytherella thrakiensis Stambolidis, 1980, RV, external, j; Mira-MAS, P1 (spr.). fig. 5. Cytherella cf. vulgata Ruggieri, 1962, RV, external; site 12 (POP 0608 ). fig. 6. Cytherelloidea cf. sordida (G. W. Müller, 1894), LV, external; Lisboa, PC-10.66-10.67. fig. 7. Bythocythere bradyi Sars, 1926, RV, external, j; MiraPMF, P2 (spr.). fig. 8. Bythocythere intermedia Elofson, 1938, LV, external, m; Lisboa, PC-22.30-22.31. fig. 9. Pseudocythere caudata Sars, 1866, RV, external,?m; Mira-MAS, P1 (spr.). fig. 10. Sclerochilus cf. abbreviatus Brady \& Robertson, 1869, RV, external,?f; Mira-MAS, P1 (aut.). fig. 11. Sclerochilus gewemuelleri Dubowsky, 1939, LV, external,?m; Mira-MAS, P1 (spr.). fig. 12. Cuneocythere semipunctata (Brady, 1868), RV, external, m; Mira-MAS, P2 (aut.). fig. 13. Pontocythere elongata (Brady, 1868), RV, external, m; Pederneira, S2-3.98. fig. 14. Cyprideis torosa (Jones, 1850), RV, external, m; Melides, MI-8.765. fig. 15. Cytheroma variabilis G. W. Müller, 1894, C, right view; Mira-MAS, P1 (spr.). fig. 16. Cytheropteron depressum Brady \& Norman, 1889, LV, external, j; Mira-MAS, P1 (aut.). fig. 17. Cytheropteron dorsocostatum Whatley \& Masson, 1980, LV, external, Lima-NSR, P1 (spr.). fig. 18. Cytheropteron latum G. W. Müller, 1894, RV, external, m; Lisboa, PC-8.15-8.16. fig. 19. Cytheropteron monoceros Bonaduce, Ciampo \& Masoli, 1976, LV, external; site 12 (POP 0108). fig. 20. Cytheropteron punctatum Brady, 1868, LV, external; site 23 (POP 0108 ). fig. 21. Cytheropteron aff. punctatum Brady, 1868, LV, external; site 11 (POP 0608). 

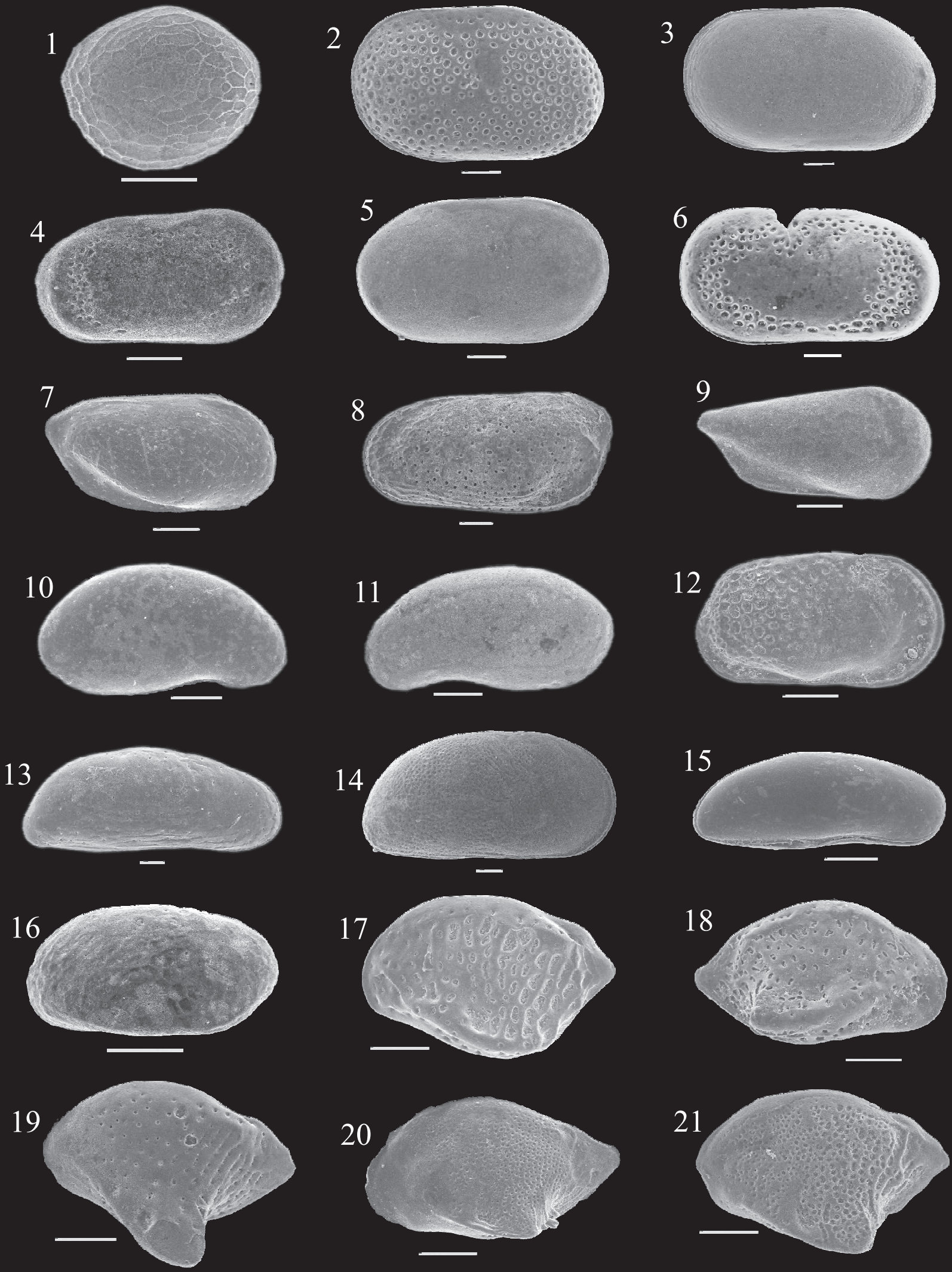
Genus Cuneocythere Lienenklaus, 1894

Cuneocythere semipunctata (Brady, 1868) - continental shelf, Mira, Holocene Lisboa, Pederneira (Pl. 1, fig. 12)

Family Cushmanideidae Puri, 1974

Genus Pontocythere Dubowsky, 1939

Pontocythere elongata (Brady, 1868) - Lima, Mira, Holocene Lisboa, Melides, Pederneira, Santo André (Pl. 1, fig. 13)

Family Cytherideidae Sars, 1925

Genus Cyprideis Jones, 1857

Cyprideis torosa (Jones, 1850) - Lima, Tejo (alive), Laje, Sado (alive), Mira, Holocene Lisboa, Melides, Pederneira, Santo André, Seixal, Sizandro (Pl. 1, fig. 14)

Family Cytheromatidae Elofson, 1939

Genus Cytheroma G. W. Müller, 1894

Cytheroma variabilis G. W. Müller, 1894 - continental shelf, Mira, Holocene Lisboa (Pl. 1, fig. 15)

Family Cytheruridae G. W. Müller, 1894

Genus Cytheropteron Sars, 1866

Cytheropteron depressum Brady \& Norman, 1889 - Mira,

Holocene Lisboa, Melides, Pederneira (Pl. 1, fig. 16)

Cytheropteron dorsocostatum Whatley \& Masson, 1980 - continental shelf, Lima, Mira, Holocene Lisboa, Pederneira (Pl. 1, fig. 17)

Cytheropteron latum G. W. Müller, 1894 - continental shelf and slope, Mira, Holocene Lisboa (Pl. 1, fig. 18)

Cytheropteron monoceros Bonaduce, Ciampo \& Masoli, 1976 continental shelf and slope (P1. 1, fig. 19)

Cytheropteron punctatum Brady, 1868 - continental slope (Pl. 1, fig. 20)

Cytheropteron aff. punctatum Brady, 1868 - continental slope (P1. 1, fig. 21)

Cytheropteron cf. ruggierii Pucci, 1956 sensu Bonaduce, Ciampo \& Masoli, 1976 - continental shelf and slope (P1. 2, fig. 1)

Cytheropteron sulcatum Bonaduce, Ciampo \& Masoli, 1976 continental shelf (Pl. 2, fig. 2)

Cytheropteron testudo Sars, 1869 - continental slope (P1. 2, fig. 3)

Cytheropteron vespertilio (Reuss, 1850) - continental shelf, Mira (P1. 2, fig. 4)

Cytheropteron volantium Whatley \& Masson, 1980 - continental shelf (Pl. 2, fig. 5)
Cytheropteron zinzulusae Bonaduce, Ciampo \& Masoli, 1976 continental slope (Pl. 2, fig. 6)

Genus Eucytherura G.W. Müller, 1894

Eucytherura complexa (Brady, 1866) - continental shelf, Mira, Holocene Lisboa (Pl. 2, fig. 7)

Eucytherura mistrettai Sissingh, 1972 - continental shelf, Mira, Holocene Lisboa (P1. 2, figs 8-9)

Genus Hemicytherura Elofson, 1941

Hemicytherura cellulosa (Norman, 1865) - continental shelf, Lima, Holocene Lisboa (P1. 2, figs 10-11)

Hemicytherura defiorei Ruggieri, 1953 - continental shelf, Mira (Pl. 2, fig. 12)

Hemicytherura aff. defiorei Ruggieri, 1953 - Mira, Holocene

Melides, Pederneira (P1. 2, fig. 13)

Hemicytherura hoskini Horne, 1981 - continental shelf, Lima, Mira, Holocene Pederneira (Pl. 2, fig. 14)

Hemicytherura videns (G. W. Müller, 1894) - continental shelf, Lima, Mira (alive), Holocene Pederneira (Pl. 2, fig. 15)

Genus Microcytherura G. W. Müller, 1894

Microcytherura fulva (Brady \& Robertson, 1874) - continental shelf and slope, Lima, Mira, Holocene Lisboa, Melides, Pederneira (Pl. 2, fig. 16)

Microcytherura cf. nigrescens G. W. Müller, 1894 - Mira (Pl. 2, fig. 17)

Genus Pseudocytherura Dubowsky, 1939

Pseudocytherura calcarata (Seguenza, 1880) - Mira, Holocene Lisboa (P1. 2, fig. 18)

Genus Semicytherura Wagner, 1957

Semicytherura acuminata (G. W. Müller, 1894) - continental shelf, Mira, Holocene Lisboa (Pl. 2, fig. 19)

Semicytherura acuta (G. W. Müller, 1912) - continental shelf, Mira, Holocene Lisboa, Melides (Pl. 2, fig. 20)

Semicytherura acuticostata (Sars, 1866) - Mira, Holocene Lisboa (P1. 2, fig. 21)

Semicytherura acuticostata ventricosa (Sars, 1866) - continental slope, Mira, Holocene Lisboa, Melides, Pederneira (P1. 3, fig. 1) Semicytherura aff. alifera Ruggieri, 1959 - Mira (Pl. 3, fig. 2)

Semicytherura angulata (Brady, 1868) - Lima, Holocene Lisboa, Pederneira (Pl. 3, fig. 3)

Semicytherura aff. angulata (Brady, 1868) - continental shelf and slope, Mira, Holocene Lisboa, Melides (Pl. 3, fig. 4)

Semicytherura arcachonensis Yassini, 1969 - Lima, Mira, Holocene Lisboa, Pederneira (Pl. 3, fig. 5)

Semicytherura cornuta (Brady, 1868) - Mira (Pl. 3, fig. 6)

Explanation of Plate 2. LV, left valve; RV, right valve; C, carapace; f, female; m, male; j, juvenile; aut., autumn; spr., spring. Scale bar $100 \mu \mathrm{m}$. fig. 1. Cytherpteron cf. ruggierii Pucci, 1956 sensu Bonaduce, Ciampo \& Masoli, 1976, RV, external; site 1 (POP 0108). fig. 2. Cytheropteron sulcatum Bonaduce, Ciampo \& Masoli, 1976, RV, external; site 13 (POP 0608). fig. 3. Cytheropteron testudo Sars, 1869, LV, external; site 23 (POP 0108). fig. 4. Cytheropteron vespertilio (Reuss, 1850), LV, external; site 12 (POP 0608). fig. 5. Cytheropteron volantium Whatley \& Masson, 1980, LV, external; site 12 (POP 0108). fig. 6. Cytheropteron zinzulusae Bonaduce, Ciampo \& Masoli, 1976, LV, external; site 11 (POP 0108). fig. 7. Eucytherura complexa (Brady, 1866), RV, external; site 13 (POP 0608). figs 8, 9. Eucytherura mistrettai Sissingh, 1972, site 13 (POP 0608): 8, RV, external; 9, LV, external, j. figs 10, 11. Hemicytherura cellulosa (Norman, 1865): 10, RV, external, f; Lima-NSR, P1 (spr.); 11, C, right view, m; Mira-PMF, P1 (aut.). fig. 12. Hemicytherura defiorei Ruggieri, 1953, RV, external; Mira-PMF, P1 (spr.). fig. 13. Hemicytherura aff. defiorei Ruggieri, 1953, LV, external; Mira-MAS, P1 (aut.). fig. 14. Hemicytherura hoskini Horne, 1981, RV, external, f; Mira-PMF, P1 (aut.). fig. 15. Hemicytherura videns (G. W. Müller, 1894), RV, external, f; Mira-CBR, P2 (spr.). fig. 16. Microcytherura fulva (Brady \& Robertson, 1874), C, right view; Mira-MAS, P1 (aut.).

fig. 17. Microcytherura cf. nigrescens G. W. Müller, 1894, C, left view; Mira-PMF, P5 (aut.). fig. 18. Pseudocytherura calcarata (Seguenza, 1880), LV, external, j; Mira-MAS, P1 (spr.). fig. 19. Semicytherura acuminata (G. W. Müller, 1894), RV, external, f; Mira-MAS, P1 (spr.). fig. 20. Semicytherura acuta (G. W. Müller, 1912), RV, external; Mira-MAS, P1 (spr.). fig. 21. Semicytherura acuticostata (Sars, 1866), RV, external,?m; Mira-MAS, P1 (spr.). 

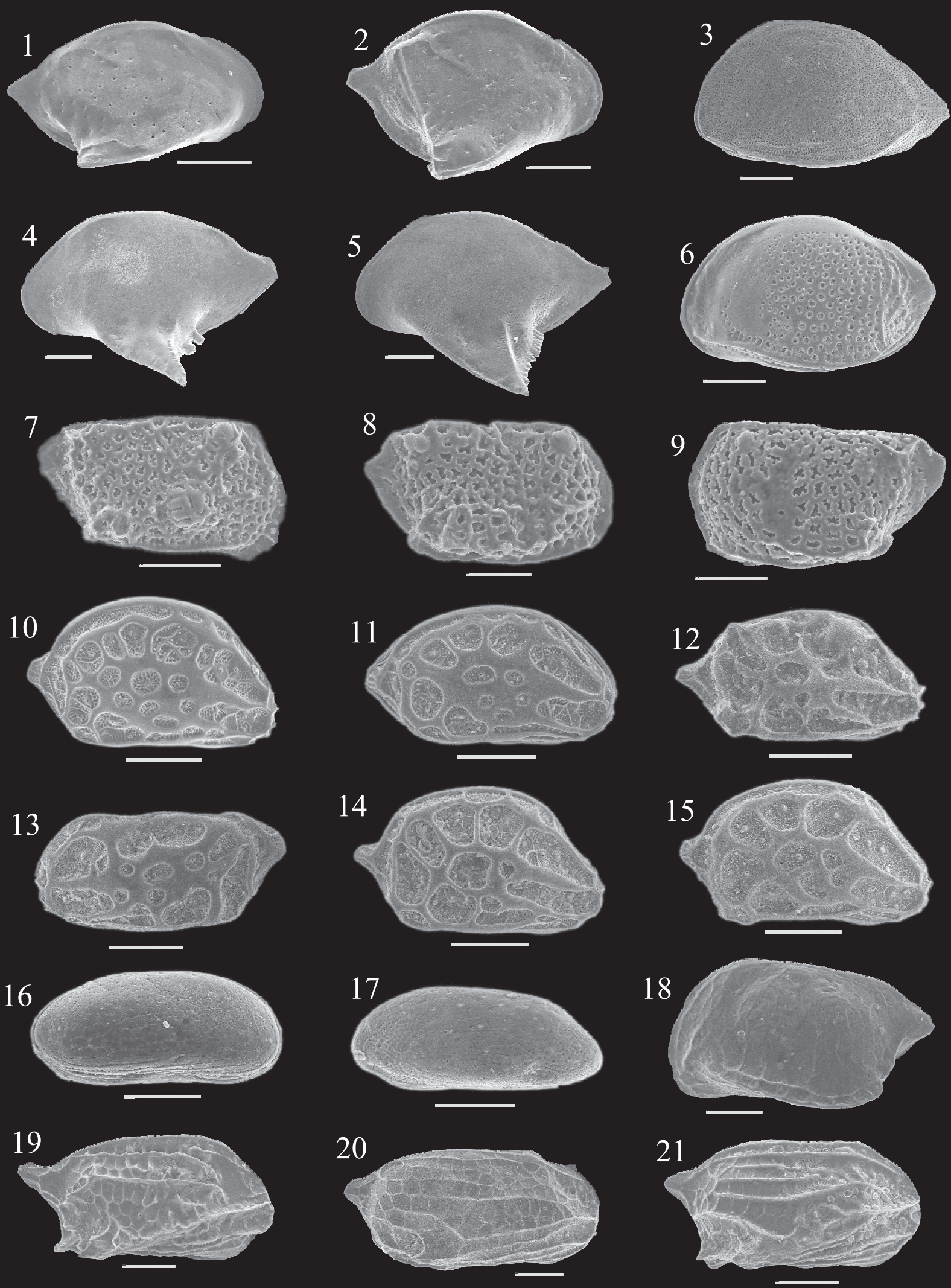

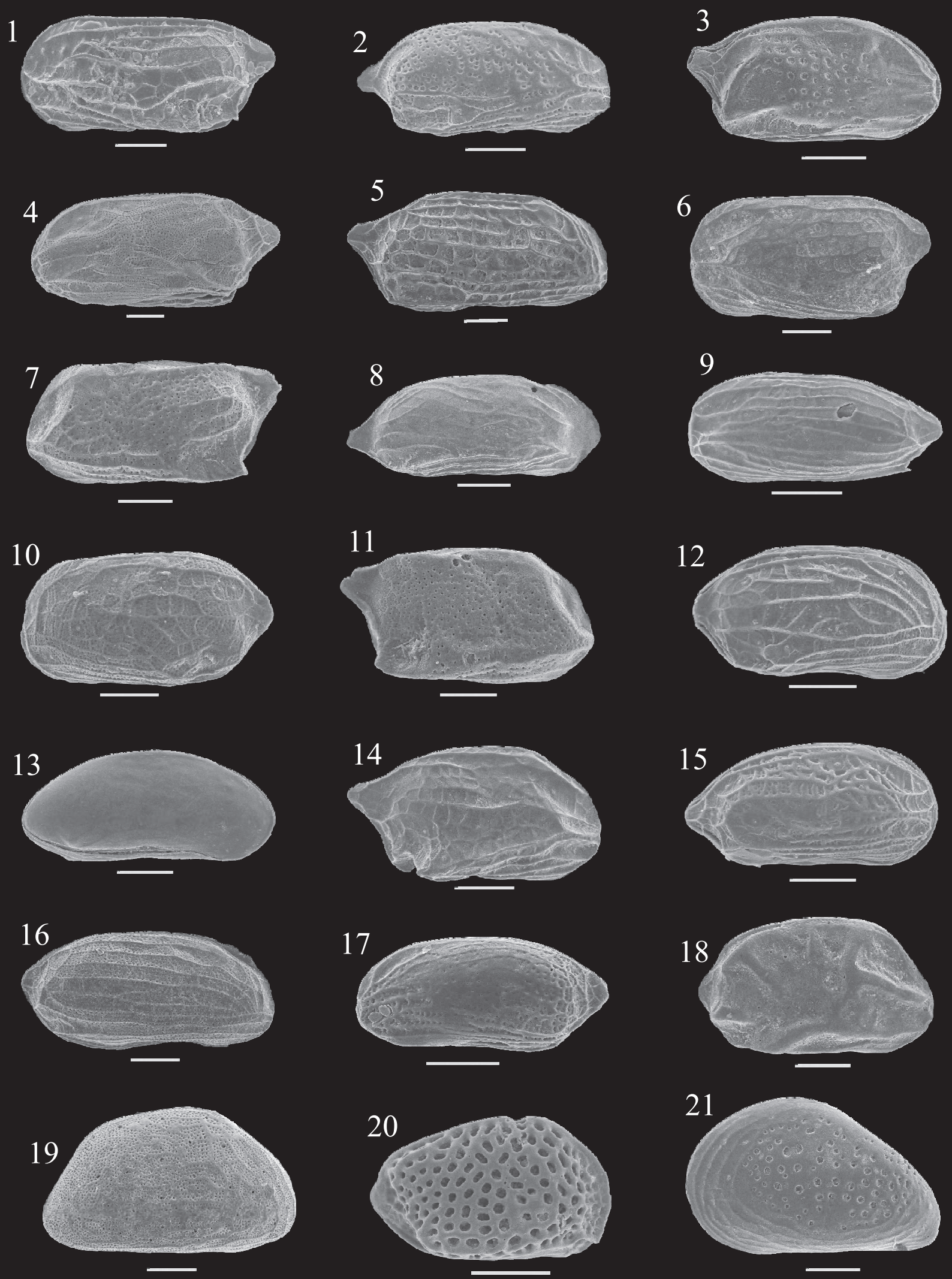
Semicytherura occulta Bonaduce, Ciampo \& Masoli, 1976 continental shelf, Holocene Lisboa (Pl. 3, fig. 7)

Semicytherura cf. producta (Brady, 1868) - Lima, Mira, Holocene Lisboa (P1. 3, fig. 8)

Semicytherura quadridentata (Hartmann, 1953) - Mira (Pl. 3, fig. 9)

Semicytherura robertsi Whittaker \& Horne, 2009 - Holocene Lisboa, Melides, Pederneira (Pl. 3, fig. 10)

Semicytherura robusta Bonaduce, Ciampo \& Masoli, 1976 continental shelf, Holocene Lisboa (Pl. 3, fig. 11)

Semicytherura sella (Sars, 1866) - Lima, Mira (alive), Holocene

Lisboa, Melides, Pederneira (Pl. 3, fig. 12)

Semicytherura simplex (Brady \& Norman, 1889) - Mira,

Holocene Lisboa, Melides (Pl. 3, fig. 13)

Semicytherura cf. stilifera Bonaduce, Ciampo \& Masoli, 1976 continental shelf, Mira, Holocene Lisboa (Pl. 3, fig. 14)

Semicytherura striata (Sars, 1866) - continental shelf, Lima,

Mira, Holocene Lisboa, Melides, Pederneira (Pl. 3, fig. 15)

Semicytherura cf. sulcata (G. W. Müller, 1894) - Mira, Holocene Lisboa, Melides (P1. 3, fig. 16)

Semicytherura tela Horne \& Whittaker, 1980 - Santo Amaro de Oeiras (rockpool, alive), Sado, Mira, Holocene Lisboa (Pl. 3, fig. 17)

Semicytherura undata (Sars, 1866) - Holocene Lisboa (P1. 3, fig. 18) Genus Tetracytherura Ruggieri, 1952

Tetracytherura angulosa (Seguenza, 1880) - continental shelf, Mira, Holocene Lisboa (Pl. 3, fig. 19)

Genus Typhlocythere Bonaduce, Ciampo \& Masoli, 1976

Typhlocythere ruggierii Bonaduce, Ciampo \& Masoli, 1976 continental slope (P1. 3, fig. 20)

Family Eucytheridae Puri, 1954

Genus Eucythere Brady, 1868

Eucythere anglica Brady, 1868 - continental shelf and slope (P1. 3, fig. 21)

Eucythere argus (Sars, 1866) - Holocene Pederneira (Pl. 4, fig. 1) Eucythere prava Brady \& Robertson, 1869 - Mira, Holocene Lisboa, Pederneira (Pl. 4, fig. 2)

Family Hemicytheridae Puri, 1953 Genus Aurila Pokorny, 1955

Aurila arborescens (Brady, 1865) - Holocene Lisboa, Melides, Pederneira, Santo André (P1. 4, fig. 3)
Aurila convexa (Baird, 1850) - continental shelf and slope, Lima, Santo Amaro de Oeiras (rockpool), Laje, Mira, Holocene Lisboa, Melides, Pederneira, Santo André (Pl. 4, fig. 4)

Aurila woutersi Horne, 1986 - Lima, Mira, Holocene Lisboa, Pederneira (Pl. 4, fig. 5)

Genus Caudites Coryell \& Fields, 1937

Caudites calceolatus (O. G. Costa, 1853) - continental shelf,

Lima, Mira, Holocene Lisboa, Pederneira (P1. 4, fig. 6)

Genus Finmarchinella Swain, 1963

Finmarchinella angulata (Sars, 1866) - Holocene Lisboa (Pl. 4, fig. 7) Genus Hemicythere Sars, 1925

Hemicythere rubida (Brady, 1868) - Holocene Lisboa (Pl. 4, fig. 8)

Hemicythere villosa (Sars, 1866) - Lima, Holocene Lisboa (Pl. 4, fig. 9)

Genus Heterocythereis Elofson, 1941

Heterocythereis albomaculata (Baird, 1838) - continental shelf,

Minho, Lima, Santo Amaro de Oeiras (rockpool, alive), Laje

(alive), Mira, Holocene Lisboa, Melides, Pederneira, Santo André (P1. 4, fig. 10)

Genus Urocythereis Ruggieri, 1950

Urocythereis britannica Athersuch, 1977 - continental shelf and slope, Lima, Sado, Mira, Holocene Lisboa, Melides, Pederneira, Santo André, Seixal (Pl. 4, fig. 11)

Family Krithidae Mandelstam, 1960

Genus Krithe Brady, Crosskey \& Robertson, 1874

Krithe keyi Breman, 1978 - continental slope (Pl. 4, fig. 12)

Krithe aff. praetexta (Sars, 1866) - continental shelf (alive) and slope (P1. 4, fig. 13)

Genus Parakrithe Van den Bold, 1958

Parakrithe dimorpha Bonaduce, Ciampo \& Masoli, 1976 continental slope (Pl. 4, fig. 14)

Genus Pseudopsammocythere Carbonnel, 1966

Pseudopsammocythere similis (G. W. Müller, 1894) - continental shelf and slope (P1. 4, fig. 15)

Family Leptocytheridae Hanai, 1957

Genus Callistocythere Ruggieri, 1953

Callistocythere badia (Norman, 1862) - Santo Amaro de Oeiras (rockpool, alive), Laje, Mira, Holocene Lisboa, Melides, Pederneira, Santo André (P1. 4, fig. 16)

Explanation of Plate 3. LV, left valve; RV, right valve; C, carapace; f, female; m, male; j, juvenile; aut., autumn; spr., spring. Scale bar $100 \mu \mathrm{m}$. fig. 1. Semicytherura acuticostata ventricosa (Sars, 1866), LV, external, m; Mira-MAS, P1 (aut.). fig. 2. Semicytherura aff. alifera Ruggieri, 1959, RV, external, m; Mira-MAS, P1 (spr.). fig. 3. Semicytherura angulata (Brady, 1868), C, right view, f; Lima-NSR, P1 (spr.). fig. 4. Semicytherura aff. angulata (Brady, 1868), C, left view,?f; Mira-PMF, P1 (aut.). fig. 5. Semicytherura arcachonensis Yassini, 1969, RV, external, m; Mira-PMF, P1 (spr.). fig. 6. Semicytherura cornuta (Brady, 1868), LV, external, f; Mira-MAS, P1 (spr.). fig. 7. Semicytherura occulta Bonaduce, Ciampo \& Masoli, 1976, LV, external,?m; Lisboa, PC-8.15-8.16. fig. 8. Semicytherura cf. producta (Brady, 1868), RV, external; Lima-NSR, P1 (spr.). fig. 9. Semicytherura quadridentata (Hartmann, 1953), C, left view; Mira-MAS, P2 (aut.). fig. 10. Semicytherura robertsi Whittaker \&Horne, 2009, LV, external, f; Pederneira, S2-3.92. fig. 11. Semicytherura robusta Bonaduce, Ciampo \& Masoli, 1976, RV, external, f; site 8 (POP 0108). fig. 12. Semicytherura sella (Sars, 1866), C, right view, f; Mira-PMF, P1 (spr.). fig. 13. Semicytherura simplex (Brady \& Norman, 1889), C, right view, m; Mira-MAS, P2 (aut.). fig. 14. Semicytherura ef. stilifera Bonaduce, Ciampo \& Masoli, 1976, RV, external, f; Mira-MAS, P1 (spr.). fig. 15. Semicytherura striata (Sars, 1866), C, right view, f; Mira-MAS, P1 (spr.). fig. 16. Semicytherura cf. sulcata (G. W. Müller, 1894), RV, external; Mira-MAS, P1 (spr.). fig. 17. Semicytherura tela Horne \& Whittaker, 1980, C, left view, m; Mira-MAS, P1 (spr.). fig. 18. Semicytherura undata (Sars, 1866), RV, external, f; Lisboa, PC-10.66-10.67. fig. 19. Tetracytherura angulosa (Seguenza, 1880), RV, external; Lisboa, PC-10.16-10.17. fig. 20. Typhlocythere ruggierii Bonaduce, Ciampo \& Masoli, 1976, RV, external; site 23 (POP 0108). fig. 21. Eucythere anglica Brady, 1868, LV, external, f; site 12 (POP 0108 ). 

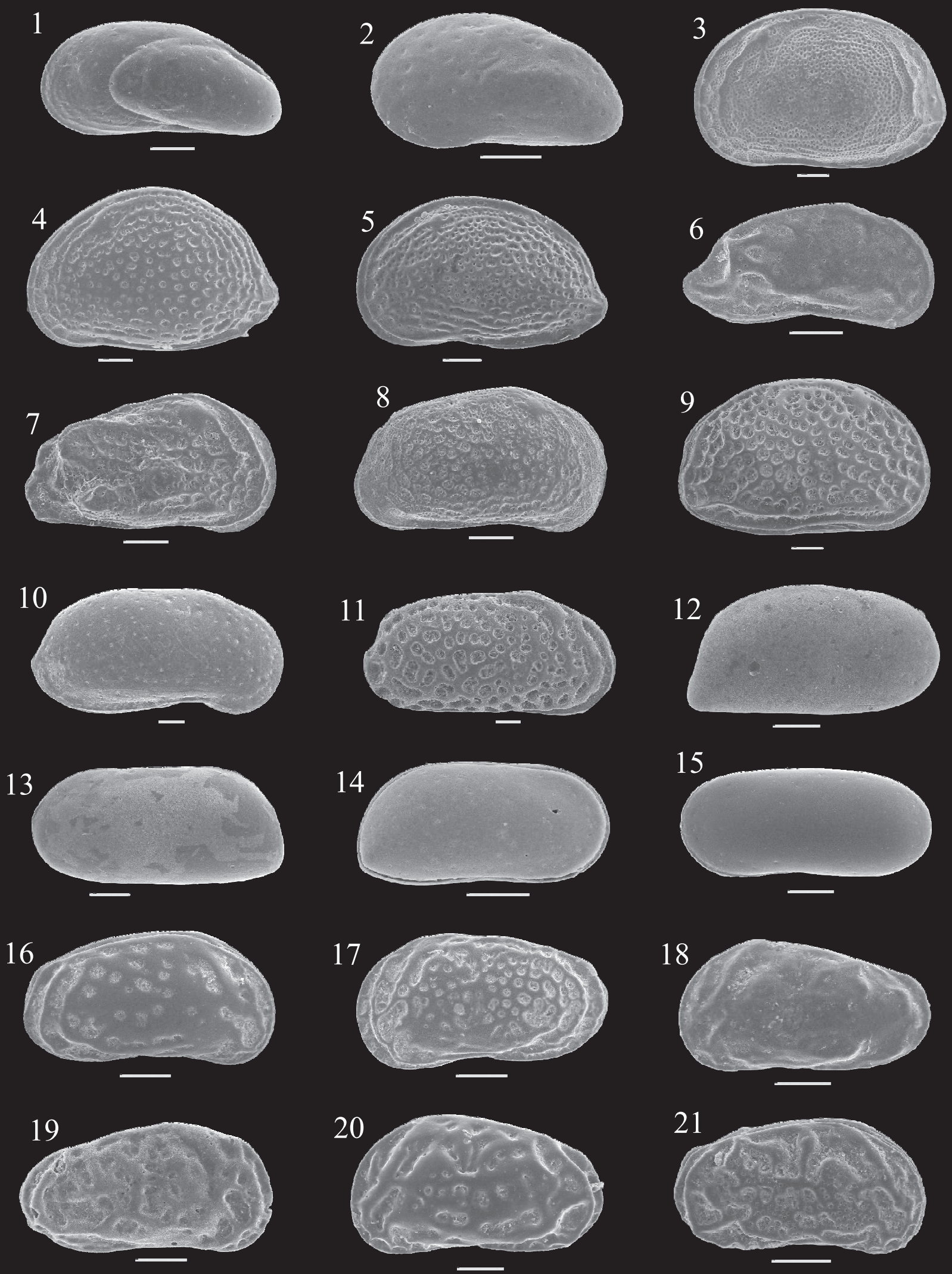
Callistocythere curryi Horne, Lord, Robinson \& Whittaker, 1990 - continental shelf and slope, Lima, Mira, Holocene Lisboa, Melides, Pederneira (Pl. 4, fig. 17)

Callistocythere diffusa (G. W. Müller, 1894) - Mira (Pl. 4, fig. 18) Callistocythere donovani Horne, Lord, Robinson \& Whittaker, 1990 - continental shelf and slope, Holocene Lisboa (P1. 4, fig. 19)

Callistocythere aff. gilva Bonaduce, Ciampo \& Masoli, 1976 Holocene Lisboa (P1. 4, fig. 20)

Callistocythere littoralis (G. W. Müller, 1894) - continental shelf, Mira, Holocene Lisboa, Melides, Pederneira (Pl. 4, fig. 21) Callistocythere lobiancoi (G. W. Müller, 1894) - Holocene Lisboa, Pederneira (Pl. 5, fig. 1)

Callistocythere murrayi Whittaker, 1978 - Minho (alive), Lima (alive), Tejo (alive), Sado, Mira, Holocene Lisboa, Seixal, Sizandro (P1. 5, fig. 2)

Genus Cluthia Neale, 1973

Cluthia keiji Neale, 1975 - continental shelf, Mira (P1. 5, fig. 3) Genus Leptocythere Sars, 1928

Leptocythere baltica Klie, 1929 - Minho (alive), Lima (alive) (P1. 5, fig. 4)

Leptocythere castanea (Sars, 1866) - Holocene Seixal (Pl. 5, fig. 5)

Leptocythere ciliata Hartmann, 1957 - Minho (alive), Lima (alive), Tejo (alive), Sado (alive), Mira (alive) (P1. 5, fig. 6) Leptocythere cribrosa (Brady, Crosskey \& Robertson, 1874) Holocene Lisboa (P1. 5, fig. 7)

Leptocythere fabaeformis (G. W. Müller, 1894) - Lima, Tejo,

Mira (alive), Holocene Lisboa, Melides, Pederneira, Santo André, Seixal (P1. 5, fig. 8)

Leptocythere lacertosa (Hirschmann, 1912) - Minho (alive), Tejo (alive), Sado (alive), Mira (alive), Holocene Lisboa, Melides, Pederneira, Santo André, Seixal (P1. 5, figs 9-10)

Leptocythere macallana (Brady \& Robertson, 1869) - Mira, Holocene Lisboa, Melides, Pederneira, Seixal (P1. 5, fig. 11) Leptocythere pellucida (Baird, 1850) - Holocene Lisboa, Pederneira, Santo André (Pl. 5, fig. 12)

Leptocythere porcellanea (Brady, 1869) - Minho (alive), Lima (alive), Tejo (alive), Sado (alive), Mira (alive), Holocene Lisboa, Pederneira, Santo André, Seixal, Sizandro (P1. 5, figs 13-14) Leptocythere psammophila Guillaume, 1976 - Minho (alive) (Pl. 5, fig. 15)

Leptocythere tenera (Brady, 1868) - continental shelf and slope, Mira, Holocene Lisboa, Pederneira (Pl. 5, fig. 16) Leptocythere sp. A - Minho (alive) (Pl. 5, figs 17-18)
Leptocythere sp. B - Minho (alive), Lima (alive), Holocene Melides (Pl. 5, fig. 19)

Family Loxoconchidae Sars, 1925

Genus Elofsonia Wagner, 1957

Elofsonia pusilla (Brady \& Robertson, 1870) - Mira, Holocene Lisboa, Melides, Pederneira (P1. 5, fig. 20)

Genus Hirschmannia Elofson, 1941

Hirschmannia viridis (O. F. Müller, 1785) - Holocene Lisboa (P1. 5, fig. 21)

Genus Loxocauda Schornikov, 1969

Loxocauda decipiens (G. W. Müller, 1894) - Mira (Pl. 6, fig. 1) Genus Loxoconcha Sars, 1866

Loxoconcha concentrica Bonaduce, Ciampo \& Masoli, 1976 continental slope (P1. 6, fig. 2)

Loxoconcha elliptica Brady, 1868 - continental shelf, Minho (alive), Lima (alive), Tejo (alive), Laje, Sado (alive), Mira

(alive), Holocene Lisboa, Melides, Pederneira, Santo André, Seixal, Sizandro (P1. 6, fig. 3)

Loxoconcha malcomsoni Horne \& Robinson, 1985 - Sado (alive), Mira (alive), Holocene Lisboa (P1. 6, fig. 4)

Loxoconcha rhomboidea (Fischer, 1855) - continental shelf and slope, Lima (alive), Tejo, Santo Amaro de Oeiras (rockpoool),

Laje, Mira (alive), Holocene Lisboa, Melides, Pederneira, Santo André, Seixal (Pl. 6, fig. 5)

Genus Nannocythere Schäfer, 1953

Nannocythere nana (Bonaduce, Ciampo \& Masoli, 1976) - Mira (Pl. 6, fig. 6)

Nannocythere pavo (Malcomson, 1886) - continental shelf, Mira (Pl. 6, fig. 7)

Genus Palmoconcha Swain \& Gilby, 1974

Palmoconcha guttata (Norman, 1865) - continental shelf and slope, Mira, Holocene Lisboa (Pl. 6, fig. 8)

Palmoconcha laevata (Norman, 1865) - continental slope, Lima, Holocene Lisboa (Pl. 6, fig. 9)

Genus Phlyctocythere Keij, 1958

Phlyctocythere pellucida (G. W. Müller, 1894) - continental shelf (Pl. 6, fig. 10)

Genus Roundstonia Neale, 1973

Roundstonia robertsoni (Brady, 1868) - Lima, Holocene Lisboa, Pederneira (Pl. 6, fig. 11)

Genus Sagmatocythere Athersuch, 1976

Sagmatocythere caelata (Ciampo, 1976) - continental shelf, Mira (P1. 6, fig. 12)

Explanation of Plate 4. LV, left valve; RV, right valve; C, carapace; f, female; m, male; j, juvenile; aut., autumn; spr., spring. Scale bar $100 \mu \mathrm{m}$ fig. 1. Eucythere argus (Sars, 1866), LV, external, m, (example of moult retention with A-1 juvenile valve attached to the adult valve); Pederneira, S2-3.98. fig. 2. Eucythere prava Brady \& Robertson, 1869, C, left view, m; Mira-MAS, P1 (spr.). fig. 3. Aurila arborescens (Brady, 1865), LV, external, f; Pederneira, S2-4.29. fig. 4. Aurila convexa (Baird, 1850), LV, external; Pederneira, S2-6.20. fig. 5. Aurila woutersi Horne, 1986, LV, external, j; Mira-MAS, P1 (aut.). fig. 6. Caudites calceolatus (O. G. Costa, 1835), RV, external; Mira-MAS, P1 (aut.). fig. 7. Finmarchinella angulata (Sars, 1866), RV, external; Lisboa, PC-21.89-21.90. fig. 8. Hemicythere rubida (Brady, 1868), RV, external, j; Lisboa, PC-24.32-24.33. fig. 9. Hemicythere villosa (Sars, 1866), $\mathrm{C}$, right view, f; Lima-NSR, P5 (aut.). fig. 10. Heterocythereis albomaculata (Baird, 1838), RV, external, m; Mira-MAS, P1 (spr.). fig. 11. Urocythereis britannica Athersuch, 1977, RV, external, m; site 12 (POP 0108). fig. 12. Krithe Keyi Breman, 1978, RV, external, f; site 23 (POP 0108 ). fig. 13. Krithe aff. praetexta (Sars, 1866), LV, external, m; site 6 (POP 0108). fig. 14. Parakrithe dimorpha Bonaduce, Ciampo \& Masoli, 1976, C, right view, f; site 23 (POP 0108). fig. 15. Pseudopsammocythere similis (G. W. Müller, 1894), C, left view; site 6 (POP 0108). fig. 16. Callistocythere badia (Norman, 1862), C, right view, f; Mira-MAS, P1 (spr.). fig. 17. Callistocythere curryi Horne, Lord, Robinson \& Whittaker, 1990, LV, external, f; Lima-NSR, P6 (spr.). fig. 18. Callistocythere diffusa (G. W. Müller, 1894), LV, external,?m; Mira-MAS, P1 (spr.). fig. 19. Callistocythere donovani Horne, Lord, Robinson \& Whittaker, 1990, RV, external, f; site 12 (POP 0608). fig. 20. Callistocythere aff. gilva Bonaduce, Ciampo \& Masoli, 1976, LV, external, f; Lisboa, PC9.66-9.67. fig. 21. Callistocythere littoralis (G. W. Müller, 1894), C, right view, f; Mira-MAS, P1 (aut.). 

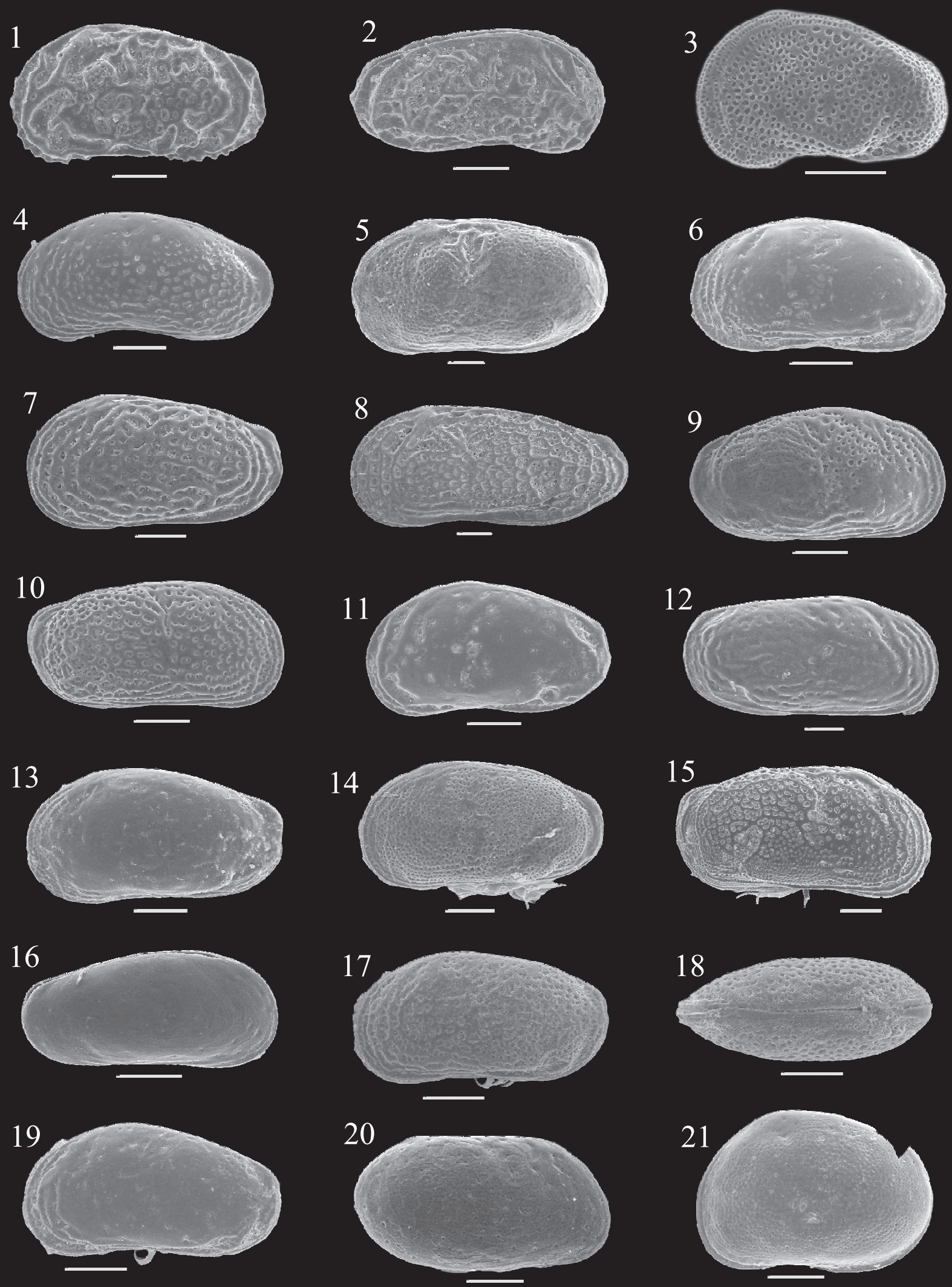
Sagmatocythere multifora (Norman, 1865) - continental shelf and slope, Holocene Lisboa (P1. 6, fig. 13)

Sagmatocythere napoliana (Puri, 1963) - Holocene Lisboa (P1. 6, fig. 14)

Sagmatocythere aff. scrupea Aiello \& Szczechura, 2004 - Mira, Holocene Lisboa (Pl. 6, figs 15-16)

Sagmatocythere variesculpta (Ruggieri, 1962) - Mira, Holocene Pederneira (Pl. 6, fig. 17)

Genus Tuberoloxoconcha Hartmann, 1973

Tuberoloxoconcha cf. atlantica Horne, 1989 - Tejo (alive), Mira (alive) (Pl. 6, figs 18-19)

Tuberoloxoconcha sp. 1 - Minho (alive) (Pl. 6, figs 20-21)

Family Neocytherideidae Puri, 1957

Genus Neocytherideis Puri, 1957

Neocytherideis subulata (Brady, 1868) - continental shelf, Lima,

Mira, Holocene Lisboa, Pederneira (Pl. 7, fig. 1)

Genus Procytherideis Ruggieri, 1978

Procytherideis cf. foveolata (G. W. Müller, 1894) - Holocene Pederneira (P1. 7, fig 2)

Procytherideis aff. subspiralis (Brady, Crosskey \& Robertson, 1874) - continental shelf, Mira, Holocene Melides (Pl. 7, fig. 3)

Genus Sahnicythere Athersuch, 1982

Sahnicythere retroflexa (Klie, 1936) - Lima, Mira, Holocene Lisboa, Pederneira (Pl. 7, fig. 4)

Family Paracytherideidae Puri, 1957

Genus Paracytheridea G. W. Müller, 1894

Paracytheridea depressa G. W. Müller, 1894 - continental shelf,

Sado, Mira, Holocene Lisboa, Melides (Pl. 7, fig. 5)

Paracytheridea triquetra (Reuss, 1850) - continental shelf, Mira, Holocene Lisboa (P1. 7, fig. 6)

Genus Tuberculocythere Colalongo \& Pasini, 1980

Tuberculocythere infelix (Bonaduce, Ciampo \& Masoli, 1976) continental shelf (Pl. 7, fig. 7)

Family Paradoxostomatidae Brady \& Norman, 1889

Genus Bradystoma Schornikov \& Keyser, 2004

Bradystoma bradyi (Sars, 1928) - Lima, Mira, Holocene Lisboa (P1. 7, fig. 8)

Genus Brunneostoma Schornikov, 1993

Brunneostoma aff. cuneatum Schornikov \& Keyser, 2004 - Mira (P1. 7, fig. 9)

Genus Cytherois G. W. Müller, 1884

Cytherois fischeri (Sars, 1866) - Minho (alive), Lima, Tejo

(alive), Sado (alive), Mira (alive), Holocene Lisboa, Melides, Pederneira, Santo André, Seixal (Pl. 7, fig. 10)
Cytherois cf. stephanidesi Klie, 1938 - Minho (alive), Lima

(alive), Tejo (alive), Holocene Melides, Pederneira (Pl. 7, fig. 11) Genus Lanceostoma Schornikov \& Keyser, 2004

Lanceostoma aff. tenerifense Schornikov \& Keyser, 2004 - Mira

(P1. 7, fig. 12)

Genus Paradoxostoma Fischer, 1855

Paradoxostoma aff. abbreviatum Sars, 1866 - Lima, Mira, Holocene Lisboa (Pl. 7, fig. 13)

Paradoxostoma cf. atrum G. W. Müller, 1894 - Mira (Pl. 7, fig. 14)

Paradoxostoma ensiforme Brady, 1868 - Lima, Mira, Holocene Lisboa, Pederneira (Pl. 7, fig. 15)

Paradoxostoma incongruens G. W. Müller, 1894 - Mira (Pl. 7, fig. 16)

Paradoxostoma robinhoodi Horne \& Whittaker, 1985 - Mira, Holocene Lisboa, Pederneira (P1. 7, fig. 17)

Paradoxostoma sarniense Brady, 1868 - Santo Amaro de Oeiras (rockpool, alive), Sado (alive), Mira (alive) (Pl. 7, fig. 18)

Paradoxostoma tenuissimum (Norman, 1869) - continental shelf (P1. 7, fig. 19)

Paradoxostoma trieri Horne \& Whittaker, 1985 - Santo Amaro de Oeiras (rockpool, alive), Sado (alive), Mira (alive) (P1. 7, fig. 20)

Paradoxostoma $\mathrm{cf}$. versicolor G. W. Müller, 1894 - Mira (Pl. 7, fig. 21)

Genus Paracytherois G. W. Müller, 1894

Paracytherois acuminata G. W. Müller, 1894 - Lima, Mira (P1. 8, fig. 1)

Paracytherois flexuosa (Brady, 1867) sensu Bonaduce, Ciampo \& Masoli, 1976 - Mira (P1. 8, figs 2-3)

Paracytherois cf. mediterranea Bonaduce, Ciampo \& Masoli, 1976 - Mira (P1. 8, fig. 4)

Family Thaerocytheridae Hazel, 1967

Genus Thaerocythere Hazel, 1967

Thaerocythere hoptonensis (Brady, Crosskey \& Robertson, 1874) - continental shelf, Lima, Santo Amaro de Oeiras (rockpool), Mira, Holocene Lisboa, Pederneira (Pl. 8, fig. 5)

Family Trachyleberididae Sylvester-Bradley, 1948 Genus Basslerites, Teichert, 1937

Basslerites teres (Brady, 1869) - continental shelf (alive), Mira (alive), Holocene Lisboa, Melides, Pederneira, Santo André (P1. 8, fig. 6)

Genus Bosquetina Keij, 1957

Bosquetina tarentina (Baird, 1850) - continental shelf (alive) (Pl. 8, fig. 7)

Genus Buntonia Howe, 1935

Explanation of Plate 5. LV, left valve; RV, right valve; C, carapace; f, female; m, male; j, juvenile; aut., autumn; spr., spring. Scale bar $100 \mu \mathrm{m}$. fig. 1. Callistocythere lobiancoi (G. W. Müller, 1894), LV, external, f; Pederneira, S3-10.84-10.85. fig. 2. Callistocythere murrayi Whittaker, 1978, C, right view, m; Mira-CBR, P1 (spr.). fig. 3. Cluthia keiji Neale, 1975, LV, external; site 1 (POP 0108). fig. 4. Leptocythere baltica Klie, 1929, C, left view, f; Lima-NSR, P1 (spr.). fig. 5. Leptocythere castanea (Sars, 1866), C, left view, f; Seixal, DWK2 285. fig. 6. Leptocythere ciliata Hartmann, 1957, C, left view, f; Lima-NSR, P6 (spr.). fig. 7. Leptocythere cribrosa (Brady, Crosskey \& Robertson, 1874), LV, external, f; Lisboa, PC-23.40-23.41. fig. 8. Leptocythere fabaeformis (G. W. Müller, 1894), C, left view, m; Mira-PMF, P1 (aut.). fig. 9. Leptocythere lacertosa (Hirschmann, 1912), RV, external, f; Pederneira, S2-3.92. fig. 10. Leptocythere lacertosa (Hirschmann, 1912), C, right view, f; Minho, Camarido-2 (spr.). fig. 11. Leptocythere macallana (Brady \& Robertson, 1869), C, left view, f; Pederneira, S2-3.92. fig. 12. Leptocythere pellucida (Baird, 1850), RV, external, f; Pederneira, S2-8.26. figs 13, 14. Leptocythere porcellanea (Brady, 1869): 13, LV, external, f; Mira-CBR, P3 (aut.); 14, C, left view, f; Minho, Camarido-2 (spr.). fig. 15. Leptocythere psammophila Guillaume, 1976, C, right view, f; Minho, Camarido-2 (spr.). fig. 16. Leptocythere tenera (Brady, 1868), C, right view, f; site 1 (POP 0108). figs 17, 18. Leptocythere sp. A, Minho-CP, P1 (spr.): 17, C, left view, f; 18, C, dorsal view, f. fig. 19. Leptocythere sp. B, C, left view, f; Minho-CP, P2 (aut.). fig. 20. Elofsonia pusilla (Brady \& Robertson, 1870), RV, external,?m; Melides, MI-9.095. fig. 21. Hirschmannia viridis (O. F. Müller, 1785), LV, external, f; Lisboa, PC-8.15-8.16. 

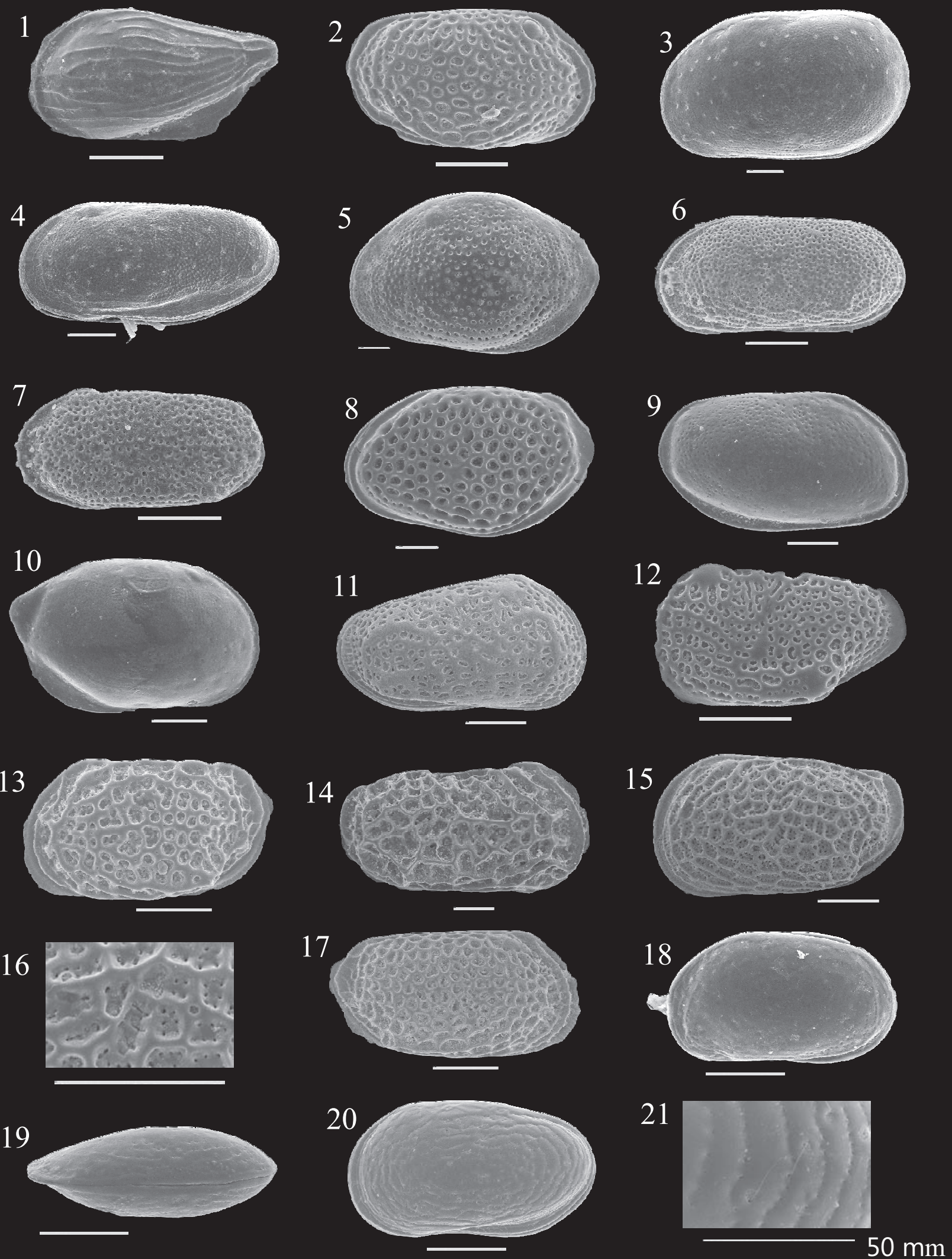
Buntonia sublatissima (Neviani, 1906) - continental shelf and slope (Pl. 8, fig. 8)

Buntonia textilis Bonaduce, Ciampo \& Masoli, 1976 - continental slope (P1. 8, fig. 9)

Genus Carinocythereis Ruggieri, 1956

Carinocythereis carinata (Roemer, 1838) - continental shelf and slope (Pl. 8, fig. 10)

Carinocythereis whitei (Baird, 1850) - continental shelf and

slope, Lima, Mira, Holocene Lisboa, Melides, Pederneira, Santo André (P1. 8, fig. 11)

Genus Celtia Neale, 1973

Celtia quadridentata (Baird, 1850) - continental shelf and slope, Mira, Holocene Pederneira (Pl. 8, fig. 12)

Genus Costa Neviani, 1928

Costa runcinata (Baird, 1850) - continental shelf and slope,

Mira, Holocene Lisboa, Pederneira (Pl. 8, fig. 13)

Genus Falunia Grekoff \& Moyes, 1955

Falunia cf. sphaerulolineata (Jones, 1857) - Mira (P1. 8, fig. 14) Genus Henryhowella Puri, 1957

Henryhowella sarsii (G. W. Müller, 1894) - continental shelf and slope (P1. 8, fig. 15)

Genus Hiltermannicythere Bassiouni, 1970

Hiltermannicythere emaciata (Brady, 1867) - Lima, Mira,

Holocene Lisboa, Melides (Pl. 8, fig. 16)

Genus Occultocythereis Howe, 1951

Occultocythereis dohrni (Puri, 1963) - Mira (Pl. 8, fig. 17)

Genus Pterygocythereis Blake, 1933

Pterygocythereis ceratoptera (Bosquet, 1852) - continental shelf (P1. 8, fig. 18)

Pterygocythereis jonesii (Baird, 1850) - continental shelf and slope, Mira, Holocene Lisboa (Pl. 8, fig. 19)

Genus Rectobuntonia Sissingh, 1972

Rectobuntonia miranda Bonaduce, Ciampo \& Masoli, 1976 continental shelf and slope (P1. 8, fig. 20)

Rectobuntonia rectangularis (Ruggieri, 1954) - continental shelf (alive) (P1. 8, fig. 21)

Family Xestoleberididae Sars, 1928

Genus Microxestoleberis G. W. Müller, 1894

Microxestoleberis nana G. W. Müller, 1894 - continental shelf, Lima, Mira (Pl. 9, fig. 1)

Microxestoleberis cf. pustulosa Ciampo, 1986 - Lima, Mira (P1. 9, fig. 2)

Genus Xestoleberis Sars, 1866
Xestoleberis labiata Brady \& Robertson, 1874 - Santo Amaro de Oeiras (rockpool, alive), Mira (alive), Holocene Lisboa, Melides, Pederneira, Santo André, Seixal (Pl. 9, fig. 3)

Xestoleberis cf. nitida (Liljeborg, 1853) - Mira (P1. 9, figs 4-5)

Xestoleberis aff. parva G. W. Müller, 1894 - continental shelf, Lima, Mira (Pl. 9, fig. 6)

Xestoleberis rubens Whittaker, 1978 - continental shelf, Lima, Sado, Mira, Holocene Lisboa, Melides, Seixal (P1. 9, fig. 7)

Superfamily Terrestricytheroidea Schornikov, 1969

Family Terrestricytheridae Schornikov, 1969

Genus Terrestricythere Schornikov, 1969

Terrestricythere aff. elisabethae Horne, Smith, Whittaker \& Murray, 2004 - Tejo (alive), Sado (alive), Mira (alive) (Pl. 9, figs 8-9)

Suborder Bairdiocopina Gründel, 1967

Superfamily Bairdioidea Sars, 1888

Family Bairdiidae Sars, 1888

Genus Bairdia McCoy, 1844

'Bairdia' subcircinata (Brady \& Norman, 1869) - continental shelf, Lima, Mira, Holocene Lisboa (P1. 9, fig. 10)

Genus Neonesidea Maddocks, 1969

Neonesidea cf. corpulenta (G. W. Müller, 1894) - Lima (P1. 9, fig. 11)

Neonesidea longevaginata (G. W. Müller, 1894) - Holocene

Lisboa, Pederneira (Pl. 9, fig. 12)

Neonesidea sp. 2 (Bonaduce, Ciampo \& Masoli, 1976) -

continental shelf, Lima, Mira, Holocene Lisboa, Pederneira (Pl. 9, fig. 13)

Genus Triebelina Van den Bold, 1946

Triebelina raripila (G. W. Müller, 1894) - Holocene Melides (P1. 9, fig. 14)

Suborder Cypridocopina Jones, 1901

Superfamily Pontocypridoidea G. W. Müller, 1894

Family Pontocyprididae G. W. Müller, 1894

Genus Argilloecia Sars, 1866

Argilloecia aff. conoidea Sars, 1923 - continental slope (Pl. 9, fig. 15)

Argilloecia minor (G. W. Müller, 1894) - continental shelf (Pl. 9, fig. 16)

Genus Pontocypris Sars, 1866

Pontocypris cf. acuminata (G. W. Müller, 1894) - continental shelf, Lima, Mira (P1. 9, fig. 17)

Explanation of Plate 6. LV, left valve; RV, right valve; C, carapace; f, female; m, male; j, juvenile; aut., autumn; spr., spring. Scale bar $100 \mu \mathrm{m}$. fig. 1. Loxocauda decipiens (G. W. Müller, 1894), LV, external; Mira-MAS, P1 (aut.). fig. 2. Loxoconcha concentrica Bonaduce, Ciampo \& Masoli, 1976, RV, external; site 11 (POP 0608). fig. 3. Loxoconcha elliptica Brady, 1868, LV, external, m; Pederneira, S2-3.92. fig. 4. Loxoconcha malcomsoni Horne \& Robinson, 1985, C, left view, m; Mira-PMF, P6 (spr.). fig. 5. Loxoconcha rhomboidea (Fischer, 1855), LV, external, f; Mira-MAS, P1 (aut.). fig. 6. Nannocythere nana (Bonaduce, Ciampo \& Masoli, 1976), LV, external; Mira-PMF, P1 (spr.). fig. 7. Nannocythere pavo (Malcomson, 1886), LV, external; Mira-PMF, P1 (aut.). fig. 8. Palmoconcha guttata (Norman, 1865), LV, external, f; site 1 (POP 0108). fig. 9. Palmoconcha laevata (Norman, 1865), RV, external, f; Lisboa, PC-21.89-21.90. fig. 10. Phlyctocythere pellucida (G. W. Müller, 1894), RV, external, f; site 13 (POP 0608) fig. 11. Roundstonia robertsoni (Brady, 1868), C, right view, m; Lima-NSR, P6 (spr.). fig. 12. Sagmatocythere caelata (Ciampo 1976), LV, external; Mira-PMF, P1 (aut.). fig. 13. Sagmatocythere multifora (Norman, 1865), LV, external, f; site 12 (POP 0608). fig. 14. Sagmatocythere napoliana (Puri, 1963), RV, external, m; Lisboa, PC-10.66-10.67. fig. 15. Sagmatocythere aff. scrupea Aiello \& Szczechura, 2004, LV, external; Mira-MAS, P1 (spr.). fig. 16. Sagmatocythere aff. scrupea Aiello \& Szczechura, 2004, LV, external, detail of ornamentation; Mira-MAS, P1 (spr.). fig. 17. Sagmatocythere variesculpta (Ruggieri, 1962), RV, external; Mira-MAS, P1 (spr.). figs 18, 19. Tuberoloxoconcha cf. atlantica Horne, 1989; Mira-PMF, P6 (spr.): 18, C, left view; 19, C, dorsal view. figs 20, 21. Tuberoloxoconcha sp. 1, C, left view, m; Minho-CP, P7A (aut.); 21, detail of ornamentation. 

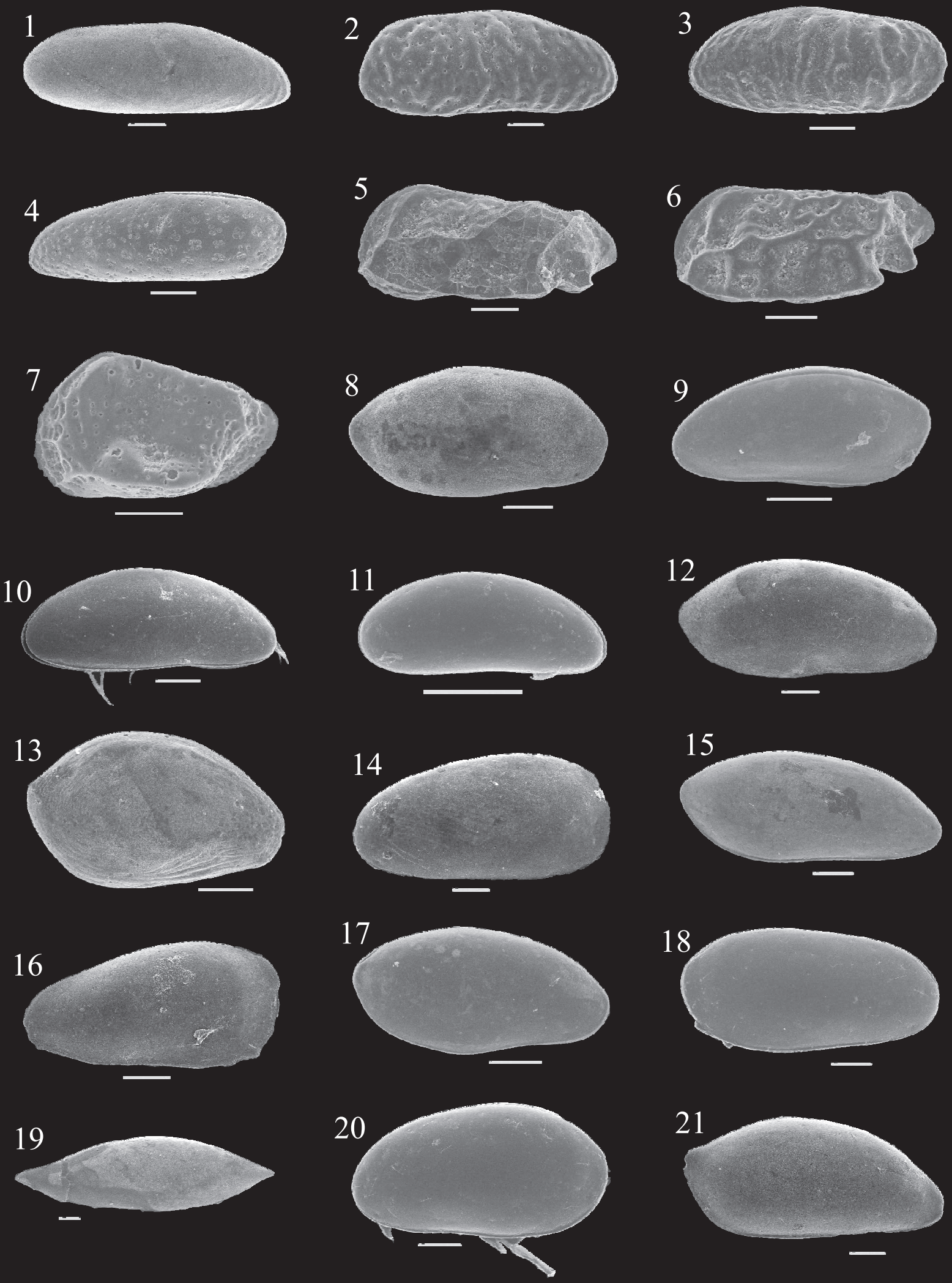
Pontocypris mytiloides (Norman, 1862) - Mira (Pl. 9, fig. 18)

Genus Propontocypris Sylvester-Bradley, 1947

Propontocypris cf. intermedia (Brady, 1868) - Mira, Holocene Lisboa, Melides, Pederneira, Santo André (P1. 9, fig. 19)

Propontocypris pirifera (G. W. Müller, 1894) - Mira, Holocene Lisboa, Pederneira (Pl. 9, fig. 20)

Superfamily Cypridoidea Baird, 1845

Family Candonidae Kaufmann, 1900

Genus Paracypris Sars, 1866

Paracypris polita Sars, 1866 - continental shelf and slope, Lima, Mira (Pl. 9, fig. 21)

\section{COMMENTS ON RECENT OSTRACODA FOUND ALIVE IN PORTUGAL}

Note that taxa found only as empty carapaces or valves are not discussed below. In addition, no mention is made of fossil records of the Recent species.

Basslerites teres (Brady, 1869)

A marine/marine to slightly brackish littoral species known from the Mediterranean to western Europe, found in France (Yassini, 1969, as Basslerites berchoni (Brady, 1870)) and England (J. Whittaker, pers. comm.). In France, Arcachon Bay, it lives at depths of 2-8m and salinity of $27 \%$ (Yassini, 1969). In Portugal found alive in the tidal flat of lower and mid-Mira estuary, in autumn and spring conditions (approximate salinity range of $32.1-33.8 \%$ and temperature $16.3-16.8^{\circ} \mathrm{C}$, in sediment interstitial water); also found alive in sites 3 (96.5 $\mathrm{m}$ depth) and 5 (94.0 $\mathrm{m}$ depth), in western Algarve continental shelf, in spring conditions.

\section{Bosquetina tarentina (Baird, 1850)}

A marine sublittoral species, known from the Mediterranean to western Europe, in France (Guillaume et al., 1985, pl. 103, figs 9-10, as Bosquetina dentata (G. W. Müller, 1894)); found living in the Mediterranean, in Bay of Bou-Ismail, Algeria, on mud and sandy mud substrates at depths of 75-220 m (Yassini, 1979, Pl. 5, figs 5-6, as Bosquetina carinella (Reuss, 1850)). In Portugal found alive in site 12 (105.3 m depth), on western Algarve continental shelf, in winter conditions.

Callistocythere badia (Norman, 1862)

A marine littoral species known from the Mediterranean to western Europe (Athersuch et al., 1989). In Portugal found alive on green and red algae, in an intertidal rockpool near Laje River mouth, Santo Amaro de Oeiras beach, in spring conditions (approximate salinity $36.2 \%$ and temperature $29.4^{\circ} \mathrm{C}$, at low tide).

Callistocythere murrayi Whittaker, 1978

A brackish species (1-8\%o at the type locality - Athersuch et al., 1989) known in western Europe, in British Isles and France (Chait et al., 1998). In Portugal found alive from the tidal flat to the low marsh in the lower Minho, mid Lima and lower Tejo estuaries, in autumn and spring conditions (approximate salinity range of $21.0-33.1 \%$ and temperature $12.4-18.5^{\circ} \mathrm{C}$, in sediment interstitial water).

Cyprideis torosa (Jones, 1850)

A common and widespread highly euryhaline species, from almost freshwater to over $60 \%$, known in Europe (as far north as Iceland), Asia, Mediterranean region, Central Africa (Athersuch et al., 1989). In Portugal found alive from the tidal flat to the high marsh in the lower Tejo and lower Sado estuaries, in autumn/ winter and spring/summer conditions (approximate salinity range of $19.0-34.1 \%$ and temperature $13.8-21.7^{\circ} \mathrm{C}$, in sediment interstitial water). It seems to prefer very large estuaries, with almost lagoonal dynamics. No noded forms have been found until now.

Cytherois fischeri (Sars, 1866)

A common and widespread brackish species, tolerating salinities from 4-35\%o, known in the Mediterranean, western Europe, to Scandinavia in the north (Athersuch et al., 1989) and Morocco in the south (Nachite et al., 2010). In Portugal found alive from the tidal flat to the lower part of the low marsh (much more abundant on the tidal flat) in the lower Minho, lower Tejo, lower Sado and lower Mira estuaries, in autumn/winter and spring conditions (approximate salinity range of $10.3-37.0 \%$ and temperature 13.7-19.7 ${ }^{\circ} \mathrm{C}$, in sediment interstitial water).

Cytherois cf. stephanidesi Klie, 1938

A brackish species found associated with algae and fine sediment, in salinities of $0-20 \%$, known in the Mediterranean and western Europe (Athersuch et al., 1989). In Portugal found alive in the lower Minho and mid Lima estuaries, from the tidal flat to the low marsh (where it is much more abundant), in autumn and spring conditions (approximate salinity range of 1.1-22.9\%o and temperature $12.4-21.4^{\circ} \mathrm{C}$, in sediment interstitial water).

Hemicytherura videns (G. W. Müller, 1894)

A marine, littoral, phytal species, known from the Mediterranean to western Europe (northern limit of its distribution appears to be in France - Yassini, 1969) and NW Morocco (Nachite et al., 2010). The Portuguese specimens which present small variation in fossal patterns, particularly in the central part,

Explanation of Plate 7. LV, left valve; RV, right valve; C, carapace; f, female; m, male; j, juvenile; aut., autumn; spr., spring. Scale bar $100 \mu \mathrm{m}$. fig. 1. Neocytherideis subulata (Brady, 1868), RV, external; Lima-NSR, P6 (spr.). fig. 2. Procytherideis cf. foveolata (G. W. Müller, 1894), RV, external; Pederneira, S2-3.98. fig. 3. Procytherideis aff. subspiralis (Brady, Crosskey \& Robertson, 1874), RV, external, j; Mira-MAS, P1 (spr.). fig. 4. Sahnicythere retroflexa (Klie, 1936), C, left view, f; Mira-MAS, P1 (spr.). fig. 5. Paracytheridea depressa G. W. Müller, 1894, LV, external,?m; MiraMAS, P1 (aut.). fig. 6. Paracytheridea triquetra (Reuss, 1850), LV, external; Mira-MAS, P1 (aut.). fig. 7. Tuberculocythere infelix (Bonaduce, Ciampo \& Masoli, 1976), LV, external; site 13 (POP 0108). fig. 8. Bradystoma bradyi (Sars, 1928), RV, external, m; Mira-PMF, P1 (aut.). fig. 9. Brunneostoma aff. cuneatum Schornikov \& Keyser, 2004, C, left view; Mira-MAS, P1 (spr.). fig. 10. Cytherois fischeri (Sars, 1866), C, right view, f; Mira-MAS, P1 (aut.). fig. 11. Cytherois cf. stephanidesi Klie, 1938, C, right view,?j; Minho-PR, P3 (aut.). fig. 12. Lanceostoma aff. tenerifense Schornikov \& Keyser, 2004, RV, external; Mira-MAS, P1 (spr.). fig. 13. Paradoxostoma aff. abbreviatum Sars, 1866, RV, external, f; Mira-MAS, P1 (spr.). fig. 14. Paradoxostoma cf. atrum G. W. Müller, 1894, LV, external; Mira-MAS, P1 (spr.). fig. 15. Paradoxostoma ensiforme Brady, 1868, RV, external,m; MiraMAS, P1 (spr.). fig. 16. Paradoxostoma incongruens G. W. Müller, 1894, LV, external; Mira-MAS, P1 (aut.). fig. 17. Paradoxostoma robinhoodi Horne \& Whittaker, 1985, RV, external, m; Mira-MAS, P1 (spr.). fig. 18. Paradoxostoma sarniense Brady, 1868 C, right view, f; Oeiras, rock pool PP2'Avm. fig. 19. Paradoxostoma tenuissimum (Norman, 1869), broken LV, external,?f; site 14 (POP 0608). fig. 20. Paradoxostoma trieri Horne \& Whittaker, 1985, C, left view, f; Oeiras, rock pool PP2'Avm. fig. 21. Paradoxostoma cf. versicolor G. W. Müller, 1894, RV, external; Mira-MAS, P1 (aut.). 

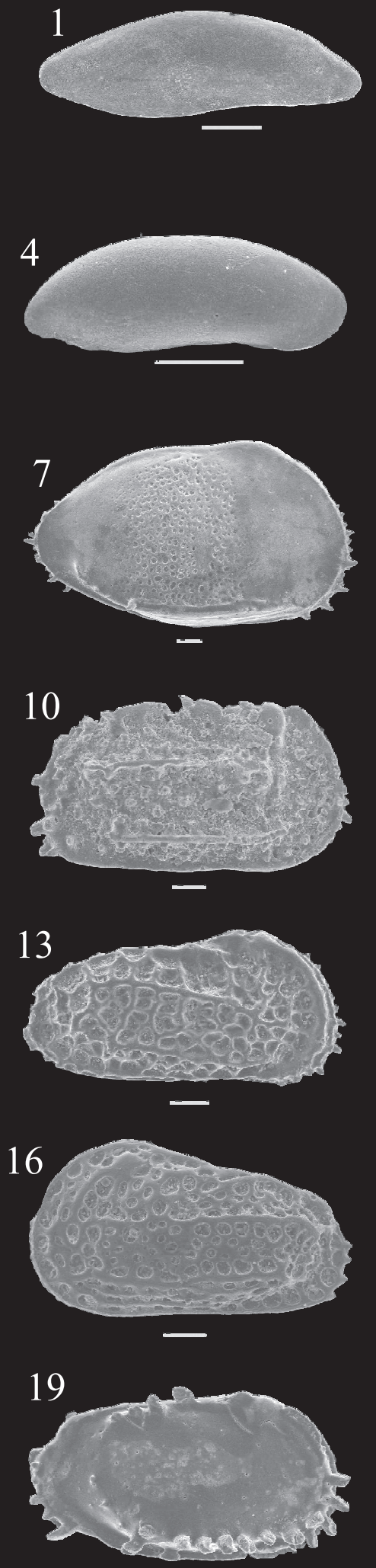
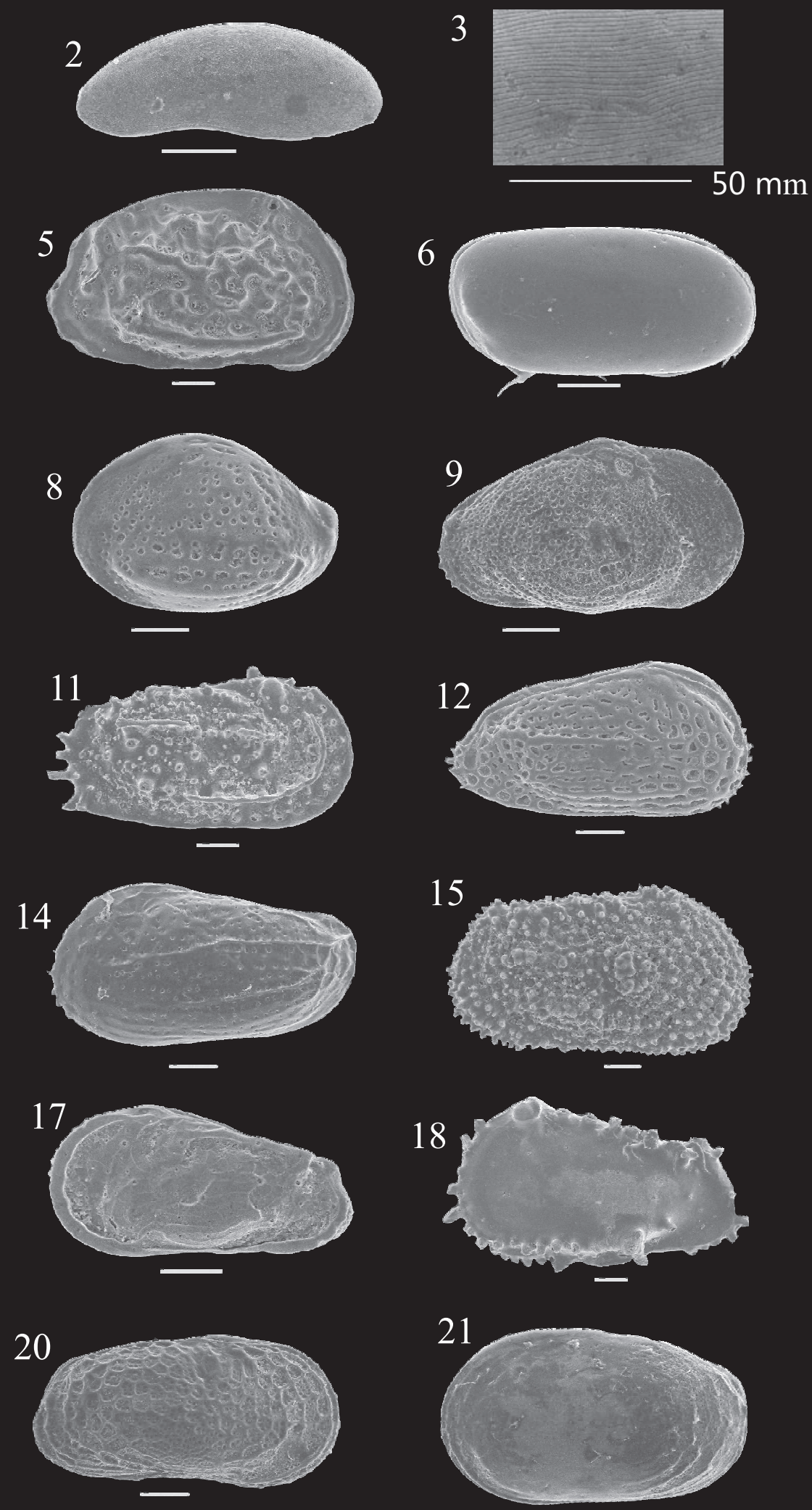
were rarely found alive in the lower and mid Mira estuary, from the tidal flat to the low marsh, in autumn and spring conditions (approximate salinity range of $23.2-42.0 \%$ and temperature $15.1-17.2^{\circ} \mathrm{C}$, in sediment interstitial water).

Heterocythereis albomaculata (Baird, 1838)

A common phytal, littoral and sublittoral marine species, often abundant in rock pools, known from the Mediterranean to northern Norway (Athersuch et al., 1989) and NW Morocco (Nachite et al., 2010). In France, Yassini (1969) reports the euryhaline nature of the species, with a salinity range of 26-34\%o. In Portugal found alive in the lowest part of Laje River estuary (Tejo's tributary), associated with green algae, in spring conditions (approximate salinity $28.9 \%$ and temperature $15.8^{\circ} \mathrm{C}$, at low tide) and in the sediment of an intertidal rock pool, in Santo Amaro de Oeiras beach (near Laje River mouth), in spring conditions (approximate salinity $36.2 \%$ and temperature $29.4^{\circ} \mathrm{C}$, at low tide).

Krithe aff. praetexta (Sars, 1866)

$K$. praetexta is a marine sublittoral species, living on sand and mud at depths of 50-500 m, from the British Isles to Sweden and Norway (Athersuch et al., 1989). The Portuguese species, closely related but with a less rounded posterior margin, was found alive in sites 5 (94.0 m depth) and 6 (100.0 m depth), western Algarve continental shelf, in spring conditions.

Leptocythere baltica Klie, 1929

A marine/brackish NW European species, occuring from the Bay of Biscay to the Baltic, usually on sand substrates, but also in British low marsh, within an approximate salinity of $25 \%$ (Horne $\&$ Boomer, 2000). In Portugal found alive in the lower Minho and lower Lima estuaries, on sand to silty sand substrates of the tidal flat and low marsh, in autumn and spring conditions (approximate salinity range of $12.6-19.7 \%$ and temperature $11.7-19.3^{\circ} \mathrm{C}$, in sediment interstitial water).

Leptocythere ciliata Hartmann, 1957

A brackish species known from Germany (North Sea), also recorded in the British Isles by Horne (1980 - unpublished $\mathrm{PhD}$ thesis) and probably in NW Morocco (Nachite et al., 2010, Pl. 2, fig. 15). In Portugal found alive in the lower Minho, lower and mid Lima, lower and mid (Trancão) Tejo, lower Sado and lower and mid Mira estuaries, from the tidal flat to the high marsh (more abundant on the low and high marsh), in autumn/winter and spring/ summer conditions (approximate salinity range of $2.5-47.5 \%$ and temperature $10.2-23.0^{\circ} \mathrm{C}$, in sediment interstitial water).

Leptocythere fabaeformis (G. W. Müller, 1894)

A brackish (highly euryhaline - 13-33\%o), phytal/littoral marine species, known from the Mediterranean to western Europe, whose northern limit of distribution is apparently in France (Yassini, 1969). In Portugal found alive only in the tidal flat of the lower Mira estuary, as subordinate species, in autumn and spring conditions (approximate salinity 33.6\% and temperature $16.3^{\circ} \mathrm{C}$, in sediment interstitial water).

Leptocythere lacertosa (Hirschmann, 1912)

A common NW European brackish species usually inhabiting estuaries on mud or fine sand substrate; its geographical distribution spans from the Baltic Sea to the NW Morocco coast (Nachite et al., 2010). In Portugal found alive from the tidal flat to the high marsh (more abundantly on the tidal flat and low marsh) of the lowest part of Minho (very rarely), lower Tejo, lower Sado and lower and mid Mira estuaries, in autumn/winter and spring/ summer conditions (approximate salinity range of $22.9-47.5 \%$ and temperature $13.6-21.7^{\circ} \mathrm{C}$, in sediment interstitial water).

Leptocythere porcellanea (Brady, 1869)

A brackish NW European species usually living on mud substrates in estuaries and salt marshes from the Baltic to SW France (Horne \& Boomer, 2000) and SW Spain (Ruiz et al., 2000). It is the most abundant species found alive in Portuguese tidal marshes of the lower Minho, mid Lima, lower and mid (Trancão) Tejo, lower Sado and lower and mid Mira estuaries, from the tidal flat to the high marsh, in autumn/winter and spring/summer conditions. It is frequently the dominant species in the low and/or in the high marsh in the SW estuaries (approximate salinity range of $2.5-47.5 \%$ and temperature $11.6-21.6^{\circ} \mathrm{C}$, in sediment interstitial water).

\section{Leptocythere psammophila Guillaume, 1976}

An outer estuarine species inhabiting sandy substrates from the Atlantic coasts of France to Iceland and the Baltic (Athersuch et al., 1989) and NW Morocco (Nachite et al., 2010); in the British Isles it was found in a low salt marsh, within a salinity range of 25-29\%o (Horne \& Boomer, 2000). In Portugal found alive on sandy to silty sand substrates of the tidal flat of the lower Minho estuary, in autumn and spring conditions (approximate salinity range of $1.1-18.6 \%$ and temperature $18.5-21.4^{\circ} \mathrm{C}$, in sediment interstitial water).

Leptocythere sp. A

Although showing affinities to some L. lacertosa in NW Europe it is best to leave this species in open nomenclature, pending further study; it is a brackish species found alive only in the lower Minho estuary, from the tidal flat to the low marsh, in autumn and spring conditions (approximate salinity range of 1.1$22.3 \%$ and temperature $13.8-21.4^{\circ} \mathrm{C}$, in sediment interstitial water).

Explanation of Plate 8. LV, left valve; RV, right valve; C, carapace; f, female; m, male; j, juvenile; aut., autumn; spr., spring. Scale bar $100 \mu \mathrm{m}$. fig. 1. Paracytherois acuminata G. W. Müller, 1894, C, right view; Mira-MAS, P1 (aut.). figs 2, 3. Paracytherois flexuosa (Brady, 1867), C, left view; Mira-PMF, P2 (spr.); 3, detail of ornamentation. fig. 4. Paracytherois cf. mediterranea Bonaduce, Ciampo \& Masoli, 1976, RV, external; Mira-PMF, P2 (aut.). fig. 5. Thaerocythere hoptonensis (Brady, Crosskey \& Robertson, 1874), RV, external; Lima-NSR, P2 (spr.). fig. 6. Basslerites teres (Brady, 1869), C, right view; site 9 (POP 0608). fig. 7. Bosquetina tarentina (Baird, 1850), RV, external, f; site 12 (POP 0108). fig. 8. Buntonia sublatissima (Neviani, 1906), LV, external; site 9 (POP 0108). fig. 9. Buntonia textilis Bonaduce, Ciampo \& Masoli, 1976, RV, external, f; site 11 (POP 0108). fig. 10. Carinocythereis carinata (Roemer, 1838), RV, external, f; site 12 (POP 0608). fig. 11. Carinocythereis whitei (Baird, 1850), RV, external,?j; site 1 (POP 0608). fig. 12. Celtia quadridentata (Baird, 1850), C, right view, f; site 12 (POP 0608). fig. 13. Costa runcinata (Baird, 1850), RV, external, m; site 3 (POP 0608). fig. 14. Falunia cf. sphaerulolineata (Jones, 1857), LV, external; Mira-MAS, P1 (aut.). fig. 15. Henryhowella sarsii (G. W. Müller, 1894); RV, external; site 9 (POP 0108). fig. 16. Hiltermannicythere emaciata (Brady, 1867), LV, external, f; Melides, MB-16.275. fig. 17. Occultocythereis dohrni (Puri, 1963), C, left view,?m; Mira-MAS, P1 (spr.). fig. 18. Pterygocythereis ceratoptera (Bosquet, 1852), LV, external, f; site 6 (POP 0108). fig. 19. Pterygocythereis jonesii (Baird, 1850), RV, external, f; site 9 (POP 0108). fig. 20. Rectobuntonia miranda Bonaduce, Ciampo \& Masoli, 1976, C, right view, m; site 11 (POP 0108). fig. 21. Rectobuntonia rectangularis (Ruggieri, 1954), LV, external; site 5 (POP 0608). 


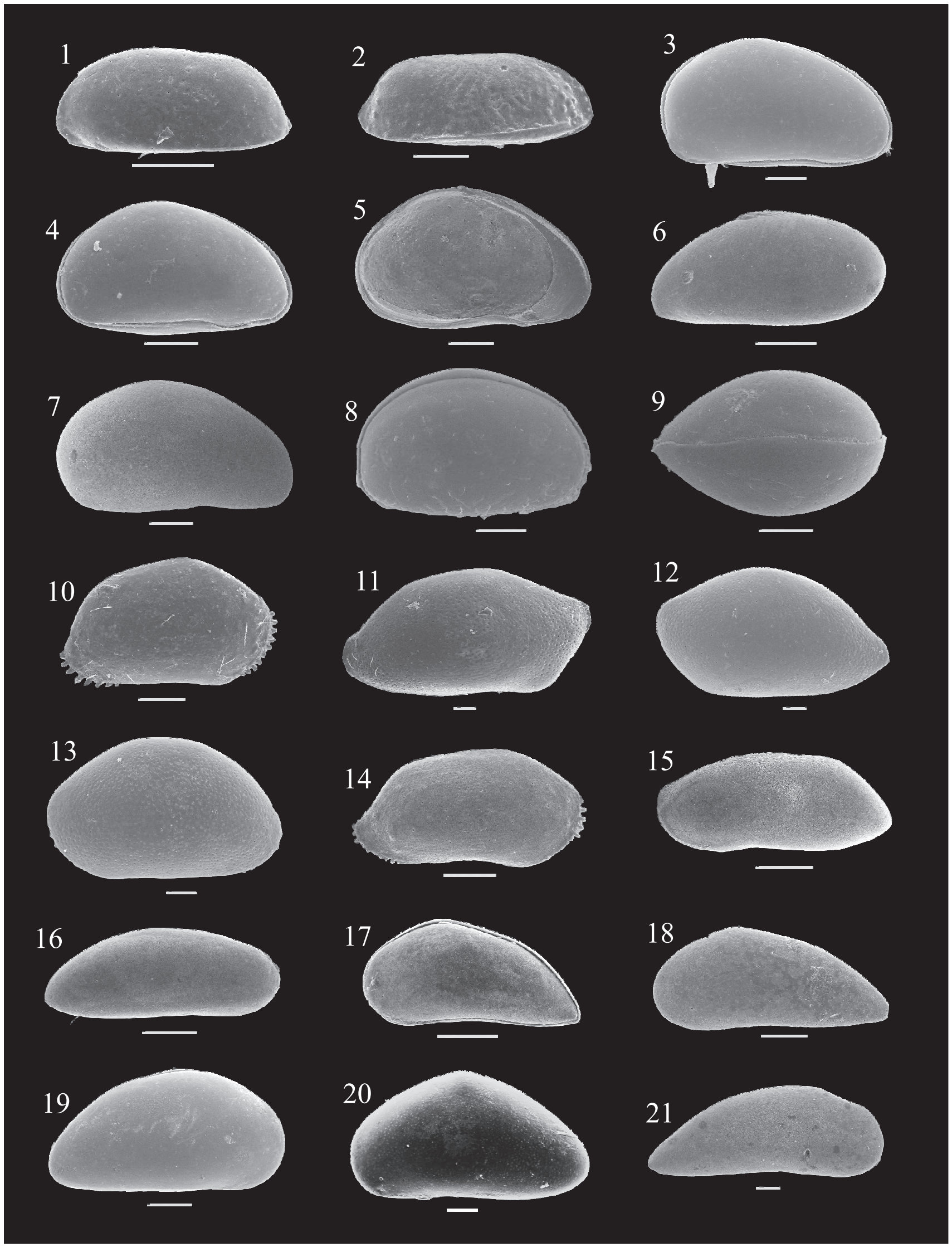


Leptocythere sp. B

Like Leptocythere sp. A, this species shows affinities to some L. lacertosa in NW Europe, but until further study it is best to leave it in open nomenclature; it is a rare brackish species found alive in the lower Minho and lower and mid Lima estuaries, from the tidal flat to the low marsh (particularly on the tidal flat), in autumn and spring conditions (approximate salinity range of 3.1$24.8 \%$ and temperature $11.6-20.2{ }^{\circ} \mathrm{C}$, in sediment interstitial water).

\section{Loxoconcha elliptica Brady, 1868}

A common brackish species usually associated with algae and mud, known from the Mediterranean to NW Europe (Horne \& Boomer, 2000) and NW Morocco (Nachite et al., 2010). In Portugal found alive in lower Minho, lower and mid Lima, lower and mid (Trancão) Tejo, lower Sado and lower and mid Mira estuaries, from the tidal flat to the high marsh, in autumn/winter and spring/summer conditions (approximate salinity range of 1.1$34.3 \%$ and temperature $10.9-21.7^{\circ} \mathrm{C}$, in sediment interstitial water).

\section{Loxoconcha malcomsoni Horne \& Robinson, 1985}

A rare brackish species, until now only known from the British Isles, living in salt marshes close to the tidal flat/low marsh limit, near marine salinity conditions (Horne \& Boomer, 2000). In Portugal found alive in the lowest part of Mira and lower Sado estuaries, from the low to the high marsh, in autumn and spring conditions (approximate salinity range of $23.2-47.5 \%$ and temperature $14.4-17.2^{\circ} \mathrm{C}$, in sediment interstitial water).

Loxoconcha rhomboidea (Fischer, 1855)

A common phytal, littoral/shallow sublittoral marine, NW European species (from Norway to Madeira and Canary Islands Athersuch et al., 1989), also found in salt marshes in slightly reduced salinities near the mouth of estuaries (Horne \& Boomer, 2000); most Mediterranean records are doubtful, but some species from Cyprus and from the western part of the Mediterranean do appear to be L. rhomboidea (Athersuch \& Whittaker, 1976). In Portugal very rarely found alive in the tidal flat of the lower Lima and lower Mira estuaries, in autumn and spring conditions (approximate salinity range of 24.8-33.8\%o and temperature 16.3$20.2^{\circ} \mathrm{C}$, in sediment interstitial water).

Paradoxostoma sarniense Brady, 1868

A phytal, marine/brackish species (tolerant to salinities as low as $25 \%$ ) apparently only known previously from the British Isles and France (Athersuch et al., 1989). In Portugal found alive on sandy substrate and red algae, in an intertidal rock pool near Laje River mouth, Santo Amaro de Oeiras beach, in spring conditions (approximate salinity range of 35.5-36.2\% and temperature 26$29.4^{\circ} \mathrm{C}$, at low tide). Also found alive in the tidal flat of the lower Sado estuary, in autumn and spring conditions (approximate salinity range of $31.6-34.1 \%$ and temperature $13.8-18.7^{\circ} \mathrm{C}$, in sediment interstitial water).

Paradoxostoma trieri Horne \& Whittaker, 1985

A phytal, littoral marine species, apparently only known previously from the British Isles and France (Horne \& Boomer, 2000); in the British Isles it also lives in salt marshes at the limit tidal flat/low marsh (Horne \& Boomer, 2000). In Portugal found alive on sandy substrate, green and red algae, in an intertidal rock pool near Laje River mouth, Santo Amaro de Oeiras beach, in spring conditions (approximate salinity range of 35.5-36.2\%o and temperature $26-29.4{ }^{\circ} \mathrm{C}$, at low tide). Also found alive in the tidal flat of lower Sado and lower Mira estuaries, in autumn and spring conditions (approximate salinity range of 33.4-34.6\%o and temperature $15.9-17.6^{\circ} \mathrm{C}$, in sediment interstitial water).

Rectobuntonia rectangularis (Ruggieri, 1954)

A marine sublittoral species known from the Mediterranean, found living at depths of 54-112 m, essentially on mud (Yassini, 1979, Pl. 4, figs 7 and 15, as Buntonia subulata Ruggieri, 1954). In Portugal found alive in sites $3(96.5 \mathrm{~m}$ depth) and $5(94.0 \mathrm{~m}$ depth), on western Algarve continental shelf, in spring conditions.

Semicytherura sella (Sars, 1866)

A shallow-marine/outer estuarine species, living on silty and sandy substrates, in NW Europe, including Norway and the Baltic Sea (Whittaker, 1974; Athersuch et al., 1989). In Portugal rarely found alive on muddy substrate in the tidal flat of the lowest part of Mira estuary, in spring conditions (approximate salinity range $32.8-35.8 \%$ and temperature $17.7-18.9^{\circ} \mathrm{C}$, in sediment interstitial water).

Semicytherura tela Horne \& Whittaker, 1980

A littoral marine/brackish species found on algae in rock pools, in British Isles and France (Athersuch et al., 1989). In Portugal, found alive (only one specimen) on sandy substrate in an intertidal rock pool near Laje River mouth, Santo Amaro de Oeiras beach, in a salinity of $35.5 \%$ and a temperature of $26^{\circ} \mathrm{C}$, at low tide, in spring conditions.

Terrestricythere aff. elisabethae Horne, Smith, Whittaker \& Murray, 2004

T. elisabethae was described in the British Isles from the high intertidal zone of a brackish creek with reeds and overhanging trees providing leaf litter (Horne et al., 2004). The Portuguese species is possibly new and was found alive only in the high marsh of lower Tejo, lower Sado and mid Mira estuaries, in

Explanation of Plate 9. LV, left valve; RV, right valve; C, carapace; f, female; m, male; j, juvenile; aut., autumn; spr., spring. Scale bar $100 \mu \mathrm{m}$. fig. 1. Microxestoleberis nana G. W. Müller, 1894, LV, external; Mira-MAS, P1 (aut.). fig. 2. Microxestoleberis cf. pustulosa Ciampo, 1986, RV, external; Mira-MAS, P1 (spr.). fig. 3. Xestoleberis labiata Brady \& Robertson, 1874, C, right view, f; Mira-MAS, P1 (aut.). figs 4, 5. Xestoleberis cf. nitida (Liljeborg, 1853): 4, C, right view, j; Mira-PMF, P1 (aut.); 5, LV, internal, f; Mira-MAS, P1 (spr.). fig. 6. Xestoleberis aff. parva G. W. Müller, 1894, LV, external; Mira- MAS, P1 (spr.). fig. 7. Xestoleberis rubens Whittaker, 1978, RV, external, f; Mira-MAS, P1 (spr.). figs 8, 9. Terrestricythere aff. elisabethae Horne, Smith, Whittaker \& Murray, 2004, C; Sado-TRO, P6 (aut.): 8, right view; 9, dorsal view. fig. 10. 'Bairdia' subcircinata (Brady \& Norman, 1869), RV, external, j; Lima-NSR, P1 (spr.). fig. 11. Neonesidea cf. corpulenta (G. W. Müller, 1894), RV, external; Lima-NSR, P3 (aut.). fig. 12. Neonesidea longevaginata (G. W. Müller, 1894), LV, external, j; Pederneira, S2-3.98. fig. 13. Neonesidea sp. 2 (Bonaduce, Ciampo \& Masoli, 1976), LV, external; Pederneira, S2-3.98. fig. 14. Triebelina raripila (G. W. Müller, 1894), RV, external, j; Melides, MB-16.915. fig. 15. Argilloecia aff. conoidea Sars, 1923, LV, external, f; site 23 (POP 0108). fig. 16. Argilloecia minor (G. W. Müller, 1894), RV, external; site 14 (POP 0108 ). fig. 17. Pontocypris cf. acuminata (G. W. Müller, 1894), C, left view; Mira-PMF, P1 (spr.). fig. 18. Pontocypris mytiloides (Norman, 1862), LV, external; MiraPMF, P1 (aut.). fig. 19. Propontocypris cf. intermedia (Brady, 1868), C, right view; Pederneira, S2-6.70. fig. 20. Propontocypris pirifera (G. W. Müller, 1894), LV, external; Lisboa, PC-18.80-18.81. fig. 21. Paracypris polita Sars, 1866, RV, external; site 23 (POP 0108). 
autumn/winter and spring/summer conditions (approximate salinity range of $18.5-25.9 \%$ and temperature of $13.4-14.4^{\circ} \mathrm{C}$, in sediment interstitial water).

Tuberoloxoconcha cf. atlantica Horne, 1989

T. atlantica is a marine, littoral, interstitial, western (Massachusetts, USA) and eastern (west Scotland) North Atlantic species (Horne, 1989). The Portuguese species, very similar but almost without ornamentation, was found alive only in the high marsh of lower Tejo and lower Mira estuaries, in autumn/winter and spring/summer conditions (approximate salinity range of $18.5-30.6 \%$ and temperature of $14.4-16.3^{\circ} \mathrm{C}$, in sediment interstitial water).

\section{Tuberoloxoconcha sp. 1}

A new brackish species found alive only in the transition zone between low and high marsh of lower Minho estuary (Coura confluence), in autumn and spring conditions, within an approximate salinity range of $10.1-22.9 \%$ and temperature of $12.9-15.4{ }^{\circ} \mathrm{C}$, in sediment interstitial water.

Xestoleberis labiata Brady \& Robertson, 1874

A shallow-marine species recorded previously only from the British Isles and France (Athersuch et al., 1989). In Portugal found alive on sandy substrate, green and red algae, in an intertidal rock pool near Laje River mouth, Santo Amaro de Oeiras beach, in spring conditions (approximate salinity range of 35.5 $36.2 \%$ and temperature $26-29.4^{\circ} \mathrm{C}$, at low tide). Also found alive in the tidal flat of the lower Mira estuary, in autumn and spring conditions (approximate salinity range of 33.4-33.8\%o and temperature $16.0-16.3^{\circ} \mathrm{C}$, in sediment interstitial water).

\section{SUMMARY}

A total of 178 brackish and marine ostracod species belonging to 75 genera has been found and identified in Portugal, most of them as empty valves. From those species, 17 are referred to as aff. or in open nomenclature; some of them are probably new. Eighteen species are recorded as fossil only and 73 have no fossil record in the area. Thirty species were found alive, most of them living in littoral areas; only 4 species were found alive in the western zone of the continental shelf of Algarve, and none was found alive in the continental slope.

\section{Autochthonous fauna}

The autochthonous fauna of the estuaries comprises 25 species, distributed in the high and low tidal marshes and in the tidal flat. Almost all the species are known in marginal-marine settings of the Atlantic coast of NW Europe - Spain, France, UK, Ireland, Belgium, The Netherlands and, for 10 of the species, even Norway, Sweden and the Baltic Sea (Cyprideis torosa, Cytherois fischeri, Heterocythereis albomaculata, Leptocythere baltica, L. lacertosa, L. porcellanea, L. psammophila, Loxoconcha elliptica, L. rhomboidea, Semicytherura sella) and also in both the Atlantic and Mediterranean coasts (9 species: Basslerites teres, Callistocythere badia, Cyprideis torosa, Cytherois fischeri, C. cf. stephanidesi, Hemicytherura videns, Heterocythereis albomaculata, Leptocythere fabaeformis, Loxoconcha elliptica). Two phytal species live in the lower estuaries, being found alive very rarely, but with very abundant empty valves in the tidal flat: Hemicytherura videns (also in the lower part of the low marsh) and Loxoconcha rhomboidea. The phytal Paradoxostoma sarniense, P. trieri and Xestoleberis labiata were found alive in the tidal flat of the SW Portuguese estuaries, in a near-marine salinity range (31.6-34.6\%). Heterocythereis albomaculata, also a phytal species, was found alive associated with green algae, always submerged (even at low tide), in the lowest part of Laje river estuary, in the confluence with Tejo, in a salinity of $28.9 \%$. Five brackish species, preferring a muddy substrate and with a wide salinity tolerance can be found in all the three environments, tidal flat, low and high marsh: Cyprideis torosa, Leptocythere ciliata, L. lacertosa, L. porcellanea and Loxoconcha elliptica.

In the tidal flat, generally muddy, where the emersion time (time of subaerial exposure between tides) ranges between 12\% and $70 \%$ and the salinity is highly variable, but no higher than $35 \%$, the number of species is high. Twenty species were found alive, from which eight are restricted to the tidal flat: L. rhomboidea, P. sarniense, P. trieri, X. labiata, Basslerites teres, Leptocythere fabaeformis, L. psammophila and Semicytherura sella. Leptocythere psammophila is associated with fine sand substrate. Cytherois fischeri is found both in the tidal flat and in the lower part of the low marsh, but it is much more abundant in the tidal flat. The most abundant species in the tidal flat are L. elliptica, $C$. fischeri, L. porcellanea and Leptocythere sp. A in the NW estuaries and C. fischeri, L. elliptica, C. torosa and L. lacertosa in the SW ones. The dominant species differs from one estuary to another and according to the location of the transect inside the estuary. In the tidal flat of the lower Lima and the lower Mira estuaries, empty valves of over 25 and 80 marine species, respectively, which do not live in these estuaries, were transported in by tidal currents.

In the low marsh, generally muddy, with emersion time around $75-90 \%$ and variable salinity (always lower in the NW estuaries, higher in the SW ones, where frequently it exceeds $35 \%$ ), fourteen species were found alive, all of them also found in the tidal flat and/or in the high marsh. The most frequent species in the low marsh are L. porcellanea, L. ciliata and L. lacertosa, present in almost all the estuaries and frequently dominant. In the NW estuaries, in addition to L. porcellanea and L. ciliata, Cytherois cf. stephanidesi, Leptocythere sp. A and L. elliptica are also well represented, particularly in the autumn. In the SW estuaries L. porcellanea and L. lacertosa are the dominant species, with $L$. ciliata, L. elliptica and C. torosa sometimes well represented. Among the other species also recorded alive in the low marsh are L. baltica and Leptocythere sp. B in the NW estuaries, L. malcomsoni in the SW ones, C. murrayi and C. fischeri in both; very rarely, Tuberoloxoconcha sp. 1 and $H$. videns occur, each one in one of the estuaries.

The high marsh, with a long emersion time (93-99.5\%) and wide range of salinity values (frequently very low, reaching $2.5 \%$ in the NW estuaries and very high, reaching $45-50 \%$ in the SW estuaries), is often barren of ostracods. Besides the five very tolerant brackish species found in some high marshes (generally in the lower part), three other species have been recorded living there: Tuberoloxoconcha sp. 1 in the NW Minho estuary, characterized by low salinities at low tide and Tuberoloxoconcha cf. atlantica and Terrestricythere aff. elisabethae in the SW estuaries. Though more abundant in the low marsh, Loxoconcha malcomsoni is also found inhabiting the lower part of the high marsh in two SW estuaries.

In the marine littoral settings, only two intertidal rock pools were studied, where six species were found living on algae or in sandy substrate, within a salinity around 35.5$36 \%$ : Callistocythere badia, Heterocythereis albomaculata, 
Paradoxostoma sarniense, P. trieri, Semicytherura tela (only in the substrate) and X. labiata.

Continental shelf and slope. On the continental shelf and slope 86 species were recognized, from which 10 were found only in the continental slope. Over $30 \%$ of the species are known, until now, both in the Atlantic Ocean and in the Mediterranean Sea, with at least 27 recorded only in the Mediterranean Sea and 19 only in the Atlantic Ocean. On the continental shelf the most common species, represented by abundant and complete populations (with all or almost all ontogenic stages, though all dead), are: Costa runcinata, Palmoconcha guttata, Krithe aff. praetexta, Pterygocythereis jonesii and Henryhowella sarsii (this species only abundant around or below $-125 \mathrm{~m}$ depth). Rectobuntonia rectangularis, Celtia quadridentata and Cytheropteron cf. ruggierii are not abundant but are frequently present. Only four species have very few specimens found alive: Basslerites teres, Bosquetina tarentina, $K$. aff. praetexta and $R$. rectangularis. B. teres is found alive as deep as $96.5 \mathrm{~m}$, but is more frequent in shallower water. In the continental slope no species were found alive. The assemblages are dominated by $K$. aff. praetexta and $H$. sarsii around $-220 \mathrm{~m}$ depth and by Paracypris polita, $K$. aff. praetexta and $K$. keyi around $-500 \mathrm{~m}$ depth.

\section{Holocene}

In the Holocene the ostracods characterize different environments and help to build a general evolutionary model of the studied marginalmarine regions since the Lateglacial (Cearreta et al., 2003; Freitas et al., 2003). This model includes several palaeoenvironmental stages, with the earliest and the latest being characterized by sediments barren in ostracods. The first stage (Lateglacial to Early Holocene), lacking ostracods, was interpreted as a high-energy fluvial environment, contemporaneous with a sea level significantly lower than present. In the second stage (Early Holocene), ostracods are present, at first represented by brackish forms (generally C. torosa and L. elliptica), progressively replaced by littoral and sublittoral marine species, the most abundant being: Urocythereis britannica, Pontocythere elongata, Basslerites teres, Carinocythereis whitei, Semicytherura robertsi, $S$. sella, X. labiata, L. rhomboidea, Aurila convexa. The maximum signal of marine influence in the sedimentary record is represented by the highest diversity in ostracods, including some deeper-water marine species, such as Celtia quadridentata, Cytheropteron dorsocostatum, C. depressum, Roundstonia robertsoni. This stage corresponds to the progressive development of an estuarine environment that further evolved to fully marine, due to the rapid sea-level rise during the Holocene transgression. A third stage (Middle and Late Holocene) is contemporaneous with a pronounced decrease in the rate of sea-level rise followed by stabilization of mean sea level. In this stage, brackish (and sometimes, rare freshwater) ostracods are once more present: C. torosa and L. elliptica if the marginal zone evolved to a barrier-lagoon system or C. torosa, L. elliptica, Cytherois fischeri, L. porcellanea, L. lacertosa (and many littoral marine/slightly brackish species) if the environment remained as an open estuary with marginal tidal marshes. The last stage (Late Holocene), lacking ostracods, corresponds to the terrestrialization of the former marginal marine systems, which became progressively shallower and smaller, due to significant input of terrestrial-sourced sediment settling in low energy depocentres and retained in alluvial fans and plains prograding and accreting over former intertidal space. In consequence, the environment resumed essentially freshwater and fluviatile conditions that persisted until present.

\section{CONCLUSIONS}

To assist both monitoring of contemporary environmental change and to assist reconstruction of Holocene palaeoenvironments, we list and figure 30 living ostracod species from a range of marginal to marine environments in Portugal. In addition, we list and figure 148 species for which we have only the calcareous carapaces or valves, some of which appear to be restricted to the Holocene and are not known living in the area.

Knowledge of Recent Portuguese ostracods is fair for the western estuarine zones, but very incomplete for the marine littoral and sublittoral zones, where only two intertidal rock pools near Lisbon and a very small area from the continental shelf and slope in south Portugal have been studied. Holocene ostracods are relatively well known in the marginal-marine settings, particularly in the lagoons.

With this work, ostracod biogeographical patterns between NW European waters and the Mediterranean Sea are clarified, with some NE Atlantic species whose southern distribution limit was set in northern Spain (Bay of Biscay) now shown to live further to the south in Portugal. Most of the listed species are typical NE Atlantic forms, especially the Recent ones, found living or accumulated in the western Portuguese estuaries, but in Algarve, in the continental shelf and slope faunas, a Mediterranean influence is clearly stronger than in the other studied environments. In future research it will be very interesting to check whether this influence is also that important in the marginal littoral Algarve assemblages.

\section{NOTE ADDED IN-PRESS}

After manuscript acceptance, further work on the western Algarve continental slope revealed 19 more Recent ostracod species and eight more genera not previously reported in the main list. All were found on the continental slope, between 230 and $513 \mathrm{~m}$ depth; only 2 species were found alive. The discoveries modify some of the manuscript data (total number of species and genera, number of live species, etc) particularly the Abstract, which are not possible to change at this stage.

Genus Monoceratina Roth, 1928 Monoceratina mediterranea Sissingh, 1971 - continental slope (alive). Genus Cytheropteron Sars, 1866 Cytheropteron aff. alatum Sars, 1866 - continental slope, Cytheropteron crassipinatum Brady \& Norman, 1888 - continental slope, Cytheropteron rotundatum Müller, 1894 - continental slope (alive). Genus Eucythere Brady, 1868 Eucythere cf. curta Ruggieri, 1975 - continental slope, Eucythere pubera Bonaduce, Ciampo \& Masoli, 1976 - continental slope. Genus Parakrithe Van den Bold, 1958 Parakrithe aff. robusta Van den Bold, 1966 - continental slope. Genus Pseudopsammocythere Carbonnel, 1966 Pseudopsammocythere aff. reniformis (Brady, 1868 ) - continental slope. Genus Loxoconchidea Bonaduce, Ciampo \& Masoli, 1975 Loxoconchidea minima Bonaduce, Ciampo \& Masoli, 1976 - continental slope. Genus Nipponocythere Ishizaki, 1971 Nipponocythere sp. 1 - continental slope. Genus Sagmatocythere Athersuch, 1976 Sagmatocythere aff. moncharmonti (Ciampo, 1971) - continental slope. Genus Echinocythereis Puri, 1953 Echinocythereis echinata (Sars, 1866) - continental slope. Genus Microxestoleberis G. W. Müller, 1894 Microxestoleberis aff. xenomys (Barbeito-Gonzàlez, 1971) - continental slope. Genus Platyleberis Bonaduce \& Danielopol, 1988 Platyleberis profunda (Breman, 1975) - continental slope. Genus Anchistrocheles Brady \& Norman, 1889 Anchistrocheles cf. tenera (Breman, 1975) - continental slope. Genus Bythocypris Brady, 1880 Bythocypris obtusata (Sars, 
1866) - continental slope. Genus Macropyxis Maddocks, 1990 Macropyxis cf. adriatica (Breman, 1975) - continental slope. Genus Argilloecia Sars, 1866 Argilloecia cf. acuminata G. W. Müller, 1894 - continental slope, Argilloecia cf. robusta Bonaduce, Ciampo \& Masoli, 1976 - continental slope.

\section{ACKNOWLEDGEMENTS}

Thanks to all authors of unpublished material used in the list, particularly Cecília Luz (also for graphical support), Florinda Monteiro, Ana Rita Figueiredo, Ana Catarina Medeiros, Rita Cardoso and Cátia Matias. Thanks go to Telmo Nunes (CBA, FCUL, Portugal) for making the SEM images and Ana Margarida Bento and Vera Lopes, both from the Centro de Geologia, FCUL, Portugal, for graphical support. Thanks also to Ana Cristina Azerêdo, Francisco Fatela, César Andrade and M. Conceição Freitas (all from the University of Lisbon, Portugal) for useful comments, David Horne (Queen Mary University of London, UK) who kindly helped in the identification of some species and Alan Lord (Senckenberg Forschungsinstitut, Germany) for encouragement and comments on a draft of the manuscript. John Whittaker (Natural History Museum, London, UK) and David Horne carefully reviewed this manuscript and provided valuable suggestions.

This paper is a contribution to Projects Storminess and Environmentally Sensitive Atlantic Coastal Areas of the European Union (ENV4-CT97-0488, 1997/2000, CeGUL - European Comission) and PRAXIS/PCNA/P/CTE/9/1996, PDCTM/P/MAR/15231/1999, POCTI/CTA/45185/2002 (MicroDyn), POCTI/MAR/55618/2004 (POPEI), PTDC/CTE-GEX/65789/2006 (PaleoNaz), PTDC/CTEGIX/105370/2008 (WesTLog), funded by the Fundação para a Ciência e Tecnologia - FCT (Portugal).

\section{Manuscript received 16 October 2012 \\ Manuscript accepted 2 November 2012 Scientific editing by Alan Lord}

\section{REFERENCES}

This section does not include all the publications in which the species listed in the text were originally described, but only references cited in the text.

Aiello, G., Barra, D., Bonaduce, G. \& Russo, A. 1996. The genus Cytherella Jones, 1849 (Ostracoda) in the Italian Tortonian-Recent. Revue de micropaléontologie, 39: 171-190.

Almeida, I.M., Andrade, C., Freitas, M.C. et al. 2009. Holocene Paleoenvironmental Evolution of the Lisbon Downtown Area as recorded in the Esteiro de Baixa Sediments - First Results. Journal of Coastal Research, Special issue, 56: 574-578.

Athersuch, J. 1976a. On Sagmatocythere napoliana (Puri). A Stereo-Atlas of Ostracod Shells, 3: 117-124.

Athersuch, J. 1976b. The genus Xestoleberis (Crustacea: ostracoda) with particular reference to Recent Mediterranean species. Publicazioni della Stazione Zoologica di Napoli, 40: 282-343.

Athersuch, J. 1981. On Hemicytherura videns (Müller). A Stereo-Atlas of Ostracod Shells, 8: 19-26.

Athersuch, J. \& Horne, D.J. 1983. On Nannocythere pavo (Malcomson). A Stereo-Atlas of Ostracod Shells, 9: 63-68.

Athersuch, J. \& Horne, D.J. 1987. Taxonomy of the genus Sclerochilus Sars (Crustacea: ostracoda) from British waters with a pictorial key. Zoological Journal of the Linnean Society, 91: 197-222.

Athersuch, J., Horne, D.J. \& Whittaker, J.E. 1989. Marine and Brackish Water Ostracods (Superfamilies Cypridacea and Cytheracea). Synopses of the British Fauna, 43. Linnean Society and Estuarine and BrackishWater Sciences Association, E.J. Brill, Leiden, 1-359.

Athersuch, J. \& Whittaker, J.E. 1976. On Loxoconcha rhomboidea (Fischer). A Stereo-Atlas of Ostracod Shells. 3: 81-90.

Athersuch, J. \& Whittaker, J.E. 1982a. On Propontocypris trigonella (Sars). A Stereo-Atlas of Ostracod Shells, 10: 29-38.

Athersuch, J. \& Whittaker, J.E. 1982b. On Propontocypris pirifera (G.W. Müller). A Stereo-Atlas of Ostracod Shells, 9: 69-76.

Barbeito-Gonzàlez, P.J. 1971. Die Ostracoden des Küstenbereiches von Naxos (Griechenland) und ihre Lebensbereiche. Mitteilungen aus dem Hamburgischen zoologischen Museum und Institut, 67: 255-326.

Bonaduce, G., Ciampo, G. \& Masoli, M. 1976. Ostracods as ecological and palaeoecological indicators. Publicazioni della Stazione Zoologica di Napoli, 40: 1-154.

Breman, E. 1975. The distribution of ostracodes in the bottom sediments of the Adriatic Sea. Vrije Universiteit Te Amsterdam, 1-165.

Cabral, M.C., Freitas, M.C., Andrade, C. \& Cruces, A. 2006. Coastal evolution and Holocene ostracods in Melides lagoon (SW Portugal). Marine Micropaleontology, 60: 181-204.

Cabral, M.C., Freitas, M.C., Andrade, C., Moreira, S. \& Cruces, A. $2011 a$. Holocene ostracods of Pederneira (Nazaré, Portugal), a structurally-segmented infilled lagoon. Joannea Geologie und Paläontologie, 11: 36-38.

Cabral, M.C., Luz, C. \& Fatela, F. 2011b. First survey of Recent ostracods from the continental shelf of Western Algarve, South Portugal. Joannea Geologie und Paläontologie, 11, 39-41.

Cearreta, A., Cachão, M., Cabral, M.C., Bao, R. \& Ramalho, M.J. 2003. Lateglacial and Holocene environmental changes in Portuguese coastal lagoons 2: microfossil multiproxy reconstruction of the Santo André coastal area. The Holocene, 13: 447-458.

Chaït, R., Dauvin, J.-C. \& Guernet, C. 1998. Les ostracodes de la Baie de Seine (Manche Orientale). Geobios, 31: 791-807.

Ciampo, G. 1976. Ostracodi pleistocenici di Cala Bianca (Marina di Camerota - Salerno). Bollettino della Società Paleontologica Italiana, 15: 3-23.

Colalongo, M.L. \& Pasini, G. 1980. La Ostracofauna plio-pleistocenica della Sezione Vrica in Calabria (con considerazioni sul limite Neogene/Quaternario). Bollettino della Società Paleontologica Italiana, 19: 44-126.

Coles, G.P., Ainsworth, N.R., Whatley, R.C. \& Jones, R.W. 1996. Foraminifera and Ostracoda from Quaternary carbonate mounds associated with gas seepage in the Porcupine Basin, offshore western Ireland. Revista Española de Micropaleontologia, 28: 113-151.

Coles, G.P., Whatley, R.C. \& Moguilevsky, A. 1994. The ostracod genus Krithe from the Tertiary and Quaternary of the North Atlantic. Palaeontology: 37, 71-120.

Doruk, N. 1974. On Triebelina raripila (G.W. Müller). A Stereo-Atlas of Ostracod Shells, 2, 65-68.

Freitas, M.C., Andrade, C., Rocha, F. et al. 2003. Lateglacial and Holocene environmental changes in Portuguese coastal lagoons: 1. The sedimentological and geochemical records of the Santo André coastal area (SW Portugal). The Holocene, 13: 433-446.

Guillaume, M.-C. 1988. On Leptocythere psammophila Guillaume. A Stereo-Atlas of Ostracod Shells, 15: 123-126.

Guillaume, M.-C., Peypouquet, J.-P. \& Tétart, J. 1985. Quaternaire et Actuel. In Oertli, H.J. (Ed.), Atlas des Ostracodes de France. Bulletin des Centres de Recherche Exploration-Production Elf-Aquitaine, Mémoire, 9: 337-377.

Hilbich, C., Mügler, .I., Daut, G., Frenzel, P., van den Borg, K. \& Mäusbacher, R. 2008. Reconstruction of the depositional history of the former coastal lagoon of Vilamoura (Algarve, Portugal): a sedimentological, microfaunal and geophysical approach. Journal of Coastal Research, 24: 83-91.

Hindson, R.A. \& Andrade, C. 1999. Sedimentation and hydrodynamic processes associated with the tsunami generated by the 1755 Lisbon earthquake. Quaternary International, 56: 27-38. 
Hindson, R.A., Andrade, C. \& Parish, R. 1999. A microfaunal and sedimentary record of environmental change within the late Holocene sediments of Boca do Rio (Algarve, Portugal). Geologie en Mijnbouw, 77: 311-321.

Horne, D.J. 1989. On Tuberoloxoconcha atlantica Horne sp. nov. A Stereo-Atlas of Ostracod Shells, 16: 73-76.

Horne, D.J. \& Boomer, I. 2000. The role of Ostracoda in saltmarsh meiofaunal communities. In Sherwood, B.R., Gardiner, B.G. \& Harris, T. (Eds), British Saltmarshes, The Linnean Society of London, Forrest Text, Cardigan, 182-202.

Horne, D.J., Cohen, A. \& Martens, K. 2002. Taxonomy, Morphology and Biology of Quaternary and Living Ostracoda. In Holmes, J.A. \& Chivas, A.R. (Eds), The Ostracoda: Applications in Quaternary Research. American Geophysical Union, Geophysical Monograph, 131: 5-36.

Horne, D.J. \& Robinson, E. 1982. On Loxoconcha cuneiformis Malcomson. A Stereo-Atlas of Ostracod Shells, 9: 21-26.

Horne, D.J. \& Robinson, E. 1985. On Loxoconcha malcomsoni Horne \& Robinson nom. nov. A Stereo-Atlas of Ostracod Shells, 12: 157.

Horne, D.J., Smith, R.J., Whittaker, J.E. \& Murray, J.W. 2004. The first British record and a new species of the superfamily Terrestricytheroidea (Crustacea, Ostracoda): morphology, ontogeny, lifestyle and phylogeny. Zoological Journal of the Linnean Society, 142: 253-288.

Horne, D.J. \& Whittaker, J.E. 1985. A revision of the genus Paradoxostoma Fischer (Crustacea; Ostracoda) in British waters. Zoological Journal of the Linnean Society, 85: 131-203.

Lachenal, A.M. 1989. Ecologie des ostracodes du Domaine Mediterraneen: application au Golfe de Gabes (Tunisie Orientale). Les variations du niveau marin depuis 30000 ans. Documents des Laboratoires de Géologie Lyon, 108: 239.

Lord, A., Cabral, M.C., Dambeck, R. \& Kunst, M. 2011. Ostracod evidence for the Neolithic environment of Rio Sizandro, Portugal. Palaeobiodiversity and Palaeoenvironments, 91: 215-228.

Loureiro, I.M., Cabral, M.C. \& Fatela, F. 2009. Marine influence in ostracod assemblages of the Mira River estuary: Comparison between lower and mid estuary tidal marsh transects. Journal of Coastal Research, Special issue, 56: 1365-1369.

Martins, M.J.F., Namiotko, T., Cabral, M.C., Fatela, F. \& Boavida, M.J. 2010. Contribution to the knowledge of the freshwater Ostracoda fauna in continental Portugal, with an updated checklist of Recent and Quaternary species. Journal of Limnology, 69: 160-173.

Nachite, D., Rodríguez-Lázaro, J., Martín-Rubio, M., Pascual, A. \& Bekkali, R. 2010. Distribution et écologie des associations d'ostracodes récents de l'estuaire de Tahadart (Maroc Nord-Occidental). Revue de micropaléontologie, 53: 3-15.

Paulo, L. 1969. Contribution à l'étude des ostracodes du Portugal. Note sur une nouvelle espèce du genre Herpetocypris. Publicações do Instituto de Zoologia Dr. A. Nobre, Fac. Ciências Porto, 108: 9-39.

Paulo, L.F. \& Moutinho, M. 1983. Systématique et distribution des ostracodes au Portugal. Publicações do Instituto de Zoologia Dr. A. Nobre, Fac. Ciencias Porto, 173: 1-32.

Ruiz, F., Gonzalez-Regalado, M.L., Baceta, J.I. \& Muñoz, J.M. 2000. Comparative ecological analysis of the ostracod faunas from low- and high-polluted southwestern Spanish estuaries: a multivariate approach. Marine Micropaleontology, 40: 345-376.

Schornikov, E.I. \& Keyser, D. 2004. The morphology and classification of Paradoxostomatinae (Ostracoda) from the nearshore zone of Madeira and the Canary Islands. Revista Española de Micropaleontologia, 36: 57-81.

van der Schriek, T., Passmore, D.G., Mugica, F.F., Stevenson, A.C., Boomer, I. \& Rolão, J. 2008. Holocene palaeoecology and floodplain evolution of the Muge tributary, Lower Tagus Basin, Portugal. Quaternary International, 189: 135-151.

Whatley, R.C. \& Masson, D.G. 1980. The Ostracod genus Cytheropteron from the Quaternary and Recent of Great Britain. Revista Española de Micropaleontologia, 11: 223-277.

Whittaker, J.E. 1974. On Semicytherura sella (Sars). A Stereo-Atlas of Ostracod Shells, 2: 85-92.

Whittaker, J.E. 1978a. On Xestoleberis nitida (Liljeborg). A Stereo-Atlas of Ostracod Shells, 5: 17-26.

Whittaker, J.E. 1978b. On Xestoleberis rubens Whittaker sp. nov. A Stereo-Atlas of Ostracod Shells, 5: 35-44.

Yassini, I. 1969. Ecologie des Associations d'Ostracodes du Bassin d'Arcachon et du littoral Atlantique. Application à l'interpretation de quelques populations du Tertiaire Aquitain. Bulletin Institut de Geologie du Bassin d'Aquitaine, 7: 1-288.

Yassini, I. 1979. The littoral system ostracodes from the Bay of Bou-Ismail, Algiers, Algeria. Revista Española de Micropaleontologia, 11: 353-416. 